\title{
Synchrotron radiation from ultrahigh-intensity laser-plasma interactions and competition with Bremsstrahlung in thin foil targets
}

\author{
B. Martinez ${ }^{*}{ }^{*}$ \\ CEA, DAM, DIF, F-91297 Arpajon, France \\ and CELIA, UMR 5107,Université de Bordeaux-CNRS-CEA, 33405 Talence, France \\ E. d'Humières (ㅁ) \\ CELIA, UMR 5107,Université de Bordeaux-CNRS-CEA, 33405 Talence, France \\ L. Gremillet ${ }^{\dagger}{ }^{\dagger}$ \\ CEA, DAM, DIF, F-91297 Arpajon, France \\ and Université Paris-Saclay, CEA, LMCE, 91680 Bruyères-le-Châtel, France
}

(Received 29 June 2020; accepted 30 October 2020; published 8 December 2020)

\begin{abstract}
The emission of high-energy photons is one of the major effects of relativistic laser-plasma interactions, which underpins a wide range of applications, from plasma diagnostics to radiography to nuclear physics and quantum electrodynamics studies. In the case of solid targets, such emission is usually dominated by Bremsstrahlung and radiative transitions of excited ions, yet one expects prolific synchrotron emission to kick in and eventually prevail at high enough laser intensities, such as those contemplated at various facilities under construction worldwide. In this paper, by means of advanced, self-consistent particle-in-cell numerical simulations, we present a detailed analysis of $\mathrm{x}$-ray and $\gamma$-ray radiation under previously unexplored interaction conditions, involving ultrathin targets partially transparent to the laser light, yet accessible to multipetawatt laser systems during their early operation phase. We first examine the characteristics of synchrotron radiation from laser-driven plasmas of varying density and size. In particular, we show and explain the dependence of the angular distribution of the radiated photons on the transparency or opacity of the plasma. We then study the competition of the synchrotron and Bremsstrahlung emissions in copper foil targets irradiated with $10^{22} \mathrm{~W} \mathrm{~cm}^{-2}, 50$-fs laser pulses. Synchrotron emission is observed to be maximized for target thicknesses of a few tens of nanometers, close to the relativistic transparency threshold, and to be superseded by Bremsstrahlung in targets a few microns thick. At their best efficiency, both mechanisms are found to radiate about $1 \%$ of the laser energy into photons with energies above $10 \mathrm{keV}$. Their energy and angular spectra are thoroughly analyzed in light of the ultrafast target expansion, the influence of which has been overlooked so far. Our results demonstrate that even using solid materials of relatively high atomic number and not-so-extreme laser pulse intensities, synchrotron radiation can be a strongly dominant and efficient source of energetic photons provided the targets are thin enough.
\end{abstract}

DOI: 10.1103/PhysRevResearch.2.043341

\section{INTRODUCTION}

The interaction of a high-intensity $\left(I_{L} \geqslant 10^{18} \mathrm{~W} \mathrm{~cm}^{-2}\right)$ laser pulse with an initially solid material sample leads to a significant fraction of the laser energy (from a few percent to $\sim 50 \%$ ) being converted into relativistic electrons [1-4]. While propagating through the dense inner region of the illuminated target (or through a secondary convertor target),

\footnotetext{
*Present address: GoLP/Instituto de Plasmas e Fusão Nuclear, Instituto Superior Técnico, Universidade de Lisboa, 1049-001 Lisbon, Portugal; bertrand.martinez@tecnico.ulisboa.pt

$\dagger$ laurent.gremillet@cea.fr

Published by the American Physical Society under the terms of the Creative Commons Attribution 4.0 International license. Further distribution of this work must maintain attribution to the author(s) and the published article's title, journal citation, and DOI.
}

these electrons can radiate part of their kinetic energy, either directly through Bremsstrahlung [5-11] or indirectly through radiative relaxation of excited atomic states [12-14]. The former process gives rise to continuous broadband photon spectra that extend up to the maximum fast electron energy, whereas the latter yields discrete spectra determined by atomic line transitions. Both types of fast-electron-induced radiation can serve for high-resolution flash radiography of dense objects [15-22], absorption spectroscopy of heated plasmas [23,24], or characterization of the fast-electron distribution [25-30]. In addition, laser-driven high-energy Bremsstrahlung photon sources have been exploited to trigger photonuclear reactions [31-35], as well as to generate unprecedented dense electronpositron pair beams through the Bethe-Heitler process in high- $Z$ thick targets [36-40].

At the extreme laser intensities $\left(I_{L} \gtrsim 10^{22} \mathrm{~W} \mathrm{~cm}^{-2}\right)$ achievable at forthcoming multipetawatt laser systems [41-45], copious emission of energetic photons can also originate from direct laser-electron interaction, that is, through 
nonlinear inverse Compton scattering of the laser light by relativistic electrons [46,47]. In the strong-field limit (such that $a_{L} \equiv e E_{L} / m_{e} c \omega_{L} \gg 1$, with $E_{L}$ the laser field strength, $\omega_{L}$ the laser frequency, $c$ the light speed, $m_{e}$ the electron mass, and $e$ the elementary charge) where the quasistationary field approximation holds, this mechanism is analogous to synchrotron emission [48-50], and its efficiency is determined by the electron quantum parameter

$$
\chi_{e}=\frac{\gamma}{E_{S}}\left[\left(\mathbf{E}_{\perp}+\mathbf{v} \times \mathbf{B}\right)^{2}+E_{\|}^{2} / \gamma^{2}\right]^{1 / 2},
$$

where $\mathbf{v}$ and $\gamma$ are the electron velocity and Lorentz factor, $(\mathbf{E}, \mathbf{B})$ is the electromagnetic field, and $E_{S}=m_{e}^{2} c^{3} / \hbar e=$ $1.3 \times 10^{18} \mathrm{~V} \mathrm{~m}^{-1}$ the Schwinger field. The subscripts $\|$ and $\perp$ denote vector components parallel or normal to $\mathbf{v}$, respectively. When $\chi_{e}$ approaches unity (specifically when $\chi_{e} \gtrsim$ 0.1 ), the average photon energy is a significant fraction of the electron kinetic energy and the emission should be treated quantum mechanically [48-50]. In this regime, the radiative losses of the laser-driven electrons can be so high as to strongly impact their dynamics and, hence, to alter the overall laser-plasma interaction [51].

Nonlinear inverse Compton/synchrotron emission can be mediated not only by the laser field but also by the strong quasistatic fields possibly induced during the laser-plasma interaction [52] or even by the self-fields of colliding, high-density electron-positron pair beams [53]. All-optical generation of $\gamma$-ray photons (with energies in the $\sim 0.1$ - to $10-\mathrm{MeV}$ range) through nonlinear inverse Compton scattering was first achieved by making a relativistic $(>100-\mathrm{MeV})$ electron beam issued from a plasma-wakefield accelerator collide with a moderately relativistic $\left(I_{L} \sim 10^{19} \mathrm{~W} \mathrm{~cm}^{-2}\right)$ femtosecond laser pulse $[54,55]$. In those pioneering experiments, however, the quantum parameter was too low $\left(\chi_{e} \lesssim 0.01\right)$ for the electron dynamics to be greatly affected by the radiation. Only recently, through the use of more intense lasers $\left(I_{L} \sim\right.$ $\left.4 \times 10^{20} \mathrm{~W} \mathrm{~cm}^{-2}\right)$ and higher-energy $(\sim 2-\mathrm{GeV})$ wakefielddriven electron beams have the first measurements of inverse Compton scattering in the radiation reaction regime $\left(\chi_{e} \gtrsim\right.$ 0.2 ) been carried out, providing evidence for substantial (up to $\sim 30 \%$ ) radiation-induced electron energy losses [56,57].

The above scenario of laser-electron-beam collisions has attracted most of the experimental interest so far, because it allows the quantum parameter to be maximized at a fixed laser intensity [58-60] and, thus, offers a promising testbed for quantum radiation reaction models [61-64] under wellcontrolled conditions. Yet this setup usually involves two synchronized powerful laser pulses (one for generating the electron beam and one for colliding with it) and so poses strong experimental constraints.

On the simulation side, moreover, most previous studies have addressed extreme-intensity $\left(I_{L} \gtrsim 10^{23-24} \mathrm{~W} \mathrm{~cm}^{-2}\right)$ interaction conditions that will be inaccessible during the early operation phases of forthcoming multi-PW systems, and hence so will be most of the high-field processes revealed by those works, such as stochasticity [65] and quantum quenching of radiation losses [66], anomalous radiative trapping [67], and pair cascading [68]. Therefore, in view of future experiments at ELI-class facilities [43], it is worthwhile to investigate the mechanisms and properties of laser-driven radiation at not-so-extreme intensities and, compared to cur- rent endeavors [56,57], in simple configurations whereby a single ultraintense laser pulse interacts with a dense plasma layer. According to previous works, significant $(\gtrsim 1 \%)$ energy conversion efficiency into high-energy radiation may be achieved at laser intensities $\gtrsim 10^{22} \mathrm{~W} \mathrm{~cm}^{-2}$ in near-criticaldensity plasmas [69-72].

In this context, it is important to determine the interaction conditions leading to synchrotron emission prevailing over Bremsstrahlung and, therefore, the scaling of the two competing radiation processes with the target parameters. This problem has as yet only been touched upon, although there is an increasing number of particle-in-cell (PIC) codes that can self-consistently describe both synchrotron radiation and Bremsstrahlung [73-78]. Notably, Pandit et al. [73] found that synchrotron emission dominates in 5- $\mu$ m-thick $\mathrm{Cu}$ targets irradiated at intensities exceeding $\sim 10^{22} \mathrm{~W} \mathrm{~cm}^{-2}$. More recently, Wan et al. [75] showed dominance of synchrotron emission at $I_{L} \geqslant 10^{21} \mathrm{~W} \mathrm{~cm}^{-2}\left(I_{L} \geqslant 10^{22} \mathrm{~W} \mathrm{~cm}^{-2}\right)$ in $1-\mu \mathrm{m}-$ thick $\mathrm{Al}(\mathrm{Au})$ targets. Still, these studies did not examine the influence of the target thickness on the radiation, with the notable exception of the study by Vyskočil et al. [76], who looked into the variations in the Bremsstrahlung spectrum from solid foils made of various materials and driven at $I_{L} \simeq 3 \times 10^{21}-10^{23} \mathrm{~W} \mathrm{~cm}^{-2}$; their investigation, however, was restricted to micrometric thicknesses, and while apparently included in their simulations, synchrotron emission was not commented upon. The same authors further examined the dynamics of synchrotron emission from solid foils in a recent work [79] which includes a brief comparison with Bremsstrahlung radiation. These previous works motivate us to further scrutinize the competition between synchrotron and Bremsstrahlung radiation in targets driven by femtosecond laser pulses, which is the main objective of this study. Special emphasis is placed on the influence of the target expansion on the radiation processes. For the first time, solid targets thin enough to enable partial transmission of the laser light are considered and shown to largely favor synchrotron emission over Bremsstrahlung. Before this, we reexamine the dependence of laser-driven synchrotron radiation on the plasma parameters. We restrict ourselves to the case of a not-so-extreme $\left(I_{L}=10^{22} \mathrm{~W} \mathrm{~cm}^{-2}\right)$ laser intensity, relevant to ELI-class facilities during their first years of operation.

This article is structured as follows. In Sec. II, we present a series of somewhat idealized PIC simulations, using planar laser waves, in order to characterize the synchrotron emission from plasmas of varying density and thickness.

These simulations are designed to give insight into the processes at play in more realistic simulations of the laserinduced radiation from copper foil targets, as reported in Sec. III. There, the Bremsstrahlung and synchrotron emissions are analyzed as a function of the target thickness and shown to strongly depend on the transparent or opaque character of the plasma. Specifically, synchrotron emission attains its maximum for target thicknesses of a few $10 \mathrm{~nm}$, close to the relativistic transparency threshold, and becomes dominated by Bremsstrahlung in targets a few micrometers thick. Our concluding remarks are gathered in Sec. IV.

\section{SYNCHROTRON EMISSION IN UNIFORM PLASMAS}

In this section, by means of two-dimensional (2D) PIC simulations, we characterize the laser-driven synchrotron 
radiation from uniform plasmas of varying density and thickness, giving rise either to in-depth penetration of the laser wave or to its absorption/reflection at the plasma boundary. Our main purpose is to identify distinct, density-dependent regimes of synchrotron emission, in the light of which the experimentally relevant, integrated simulations in Sec. III will be analyzed.

\section{A. Numerical setup and modeling}

Our simulations have been performed using the CALDER PIC code [80-82]. The laser pulse is modeled as an electromagnetic plane wave of wavelength $\lambda_{L} \equiv 2 \pi c / \omega_{L}=1 \mu \mathrm{m}$ and peak intensity $I_{L}=10^{22} \mathrm{~W} \mathrm{~cm}^{-2}\left(a_{L}=85\right)$, linearly polarized along the $y$ axis and propagating in the $+x$ direction. Unless otherwise stated, it has a constant temporal profile, preceded by a two-cycle-long (6.6-fs) linear ramp. The irradiated plasma slab is made of fully ionized carbon ions $\left(\mathrm{C}^{6+}\right)$ and electrons of uniform density profile. Introducing the critical density $n_{c} \equiv m_{e} \epsilon_{0} \omega_{L}^{2} / e^{2} \simeq 1.1 \times 10^{21} \mathrm{~cm}^{-3}\left(\epsilon_{0}\right.$ is the vacuum permittivity), the initial electron density is set to either $n_{e 0}=17 n_{c}$ or $100 n_{c}$, leading, respectively, to relativistic self-induced transparency or opacity of the plasma. The density profile is either of finite length $(l=1 \mu \mathrm{m})$ or 'semi-infinite,' i.e., long enough to prevent both the laser pulse and the accelerated particles from reaching its rear boundary over the time span of the simulations $(t \simeq 150 \mathrm{fs})$. The time origin $(t=0)$ is chosen to be when the laser peak intensity hits the (sharp) plasma front boundary, located at $x=16 \mu \mathrm{m}$.

The 2D domain comprises $4800 \times 400$ cells, of cell size $\Delta x=\Delta y=\lambda_{L} / 60$. Each cell initially contains 10 macroparticles per plasma species. The time step is $\Delta t=0.6 \Delta x$. The boundary conditions for both fields and particles are taken to be absorbing in the $x$ direction and periodic in the $y$ direction. Coulomb collisions between charged particles [83] and synchrotron radiation [81] are described. The synchrotron module implemented in CALDER combines a continuous radiation reaction model [61] for electrons with $\chi_{e} \leqslant 10^{-3}$ and a Monte Carlo quantum model [84] for electrons with $\chi_{e} \geqslant 10^{-3}$. The chosen threshold value between the two regimes is quite arbitrary yet ensures that the quantum regime is accurately captured. Bremsstrahlung is not modeled in this section. Since we do not describe the subsequent interaction of the radiated photons with the plasma particles or the electromagnetic fields, they are not advanced on the simulation grid but their properties are stored for postprocessing.

\section{B. Relativistically undercritical plasma}

We first consider the case of a semi-infinite plasma of density $n_{e 0}=17 n_{c}$. The main features of the laser-plasma interaction and ensuing high-energy radiation are illustrated at time $t=36$ fs in Figs. 1(a)-1(c). Figure 1(a) plots lineouts of the $E_{y}$ and $B_{z}$ field components (in units of $E_{0}=m_{e} c \omega_{L} / e=$ $3.2 \times 10^{12} \mathrm{~V} \mathrm{~m}^{-1}$ and $B_{0}=m_{e} \omega_{L} / e=1.1 \times 10^{4} \mathrm{~T}$, respectively) as well as of the electron density (in units of $n_{c}$ ). One can see that the laser wave has then traveled a few micrometers through the plasma (the dashed vertical curve indicates the vacuum/plasma interface). Albeit modulated by the laser ponderomotive force and the induced plasma waves,

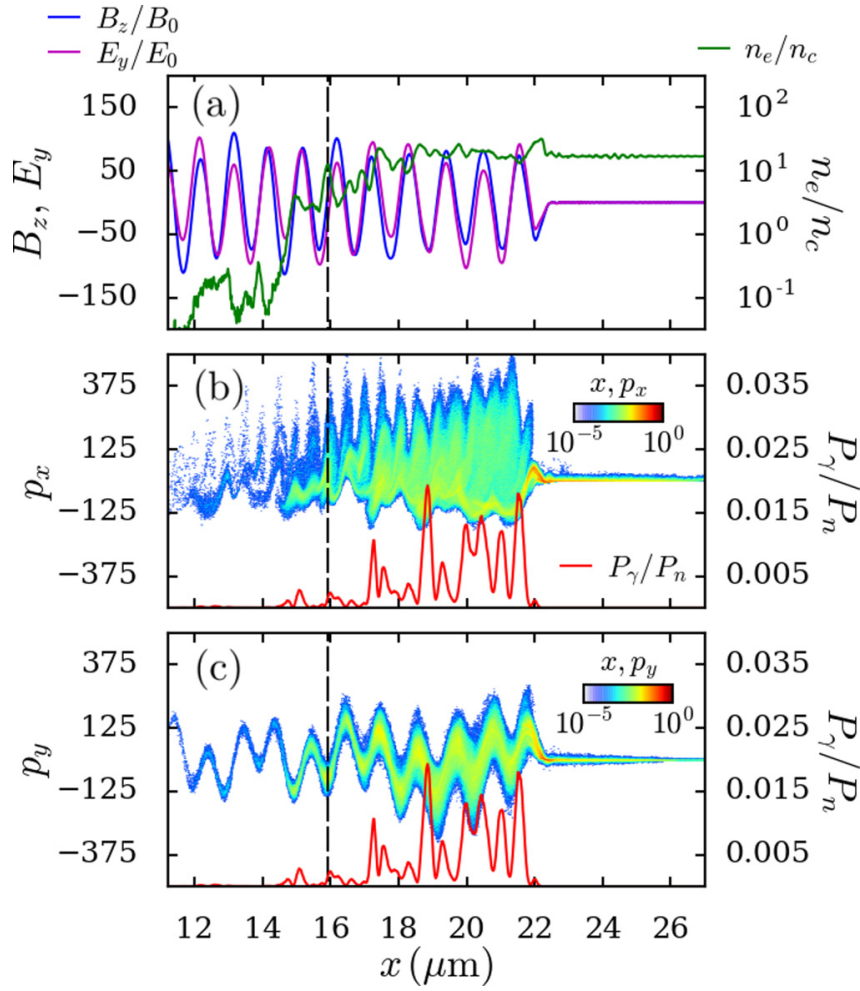

FIG. 1. Interaction of a semi-infinite, electromagnetic plane wave $\left(I_{L}=10^{22} \mathrm{~W} \mathrm{~cm}^{-2}\right)$ with a semi-infinite, relativistically undercritical $\mathrm{C}^{6+}$ plasma $\left(n_{e 0}=17 n_{c}\right)$. (a) Longitudinal lineouts of the $E_{y}$ (purple) and $B_{z}$ (blue) field components and of the electron density $n_{e}$ (green). (b) $x$ - $p_{x}$ electron phase space (averaged along $y$ ). (c) $x-p_{y}$ electron phase space (averaged over $y$ ). In (b) and (c) the red curve plots a longitudinal lineout of the synchrotron radiated power density $\left(P_{\gamma}\right)$. All quantities are recorded $36 \mathrm{fs}$ after the on-target laser peak.

the electron density profile keeps an average value close to its initial value, as expected in the relativistic self-induced transparency regime [85].

Figures 1(b) and 1(c) show the $x$ - $p_{x}$ and $x$ - $p_{y}$ electron phase spaces (both averaged along $y$ ). In each panel is overlaid the longitudinal profile of the radiated power density $P_{\gamma}$, normalized to $P_{n}=(2 / 3) \alpha_{f} n_{c} m_{e} c^{2} / \tau_{C} \simeq 3.4 \times 10^{26} \mathrm{~W} \mathrm{~cm}^{-3}\left(\alpha_{f}\right.$ is the fine-structure constant and $\tau_{C}=\hbar / m_{e} c^{2}$ the Compton time). The emission is seen to take place throughout the irradiated plasma region, in which the electrons have been accelerated to ultrarelativistic (longitudinal and transverse) momenta. The $x-p_{x}$ phase space exhibits the usual forwardmoving, high-energy (up to $p_{x} / m_{e} c \approx 500$ ) electron jets spatially modulated at $\lambda_{L} / 2$, but also a denser electron return current accelerated at $\left|p_{x}\right| / m_{e} c \approx 100-200$. Those counterstreaming electrons are first pushed forward in the rising edge of the laser wave before getting pulled back by the charge separation field, as analyzed by Debayle et al. [86]. The laser front moves at a velocity $v_{f} / c \approx 0.47$, somewhat lower than that predicted $\left(v_{f} / c \simeq 0.56\right)$ from Ref. [85], probably as a result of mobile ions that favor electron compression (up to $n_{e} \approx 40 n_{c}$ ) at the laser head. Transverse electron momenta as high as $\left|p_{y}\right| / m_{e} c \approx 300$ are shown in Fig. 1(c), which may seem surprising since one expects $\left|p_{y} /\right| m_{e} c \leqslant\left|B_{z}\right| / B_{0}$ for an electromagnetic plane wave propagating in a dissipation-free 


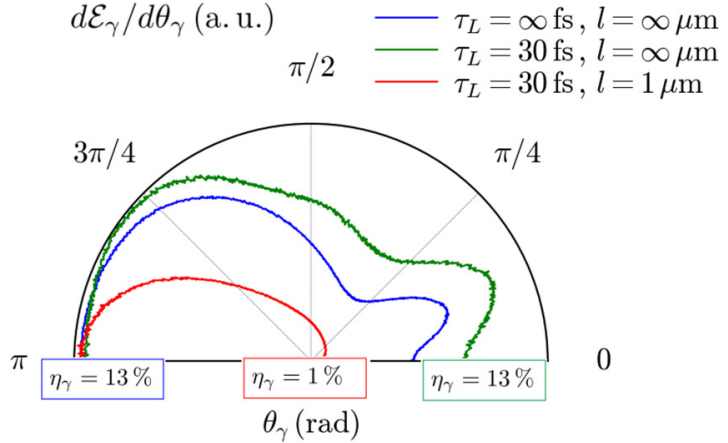

FIG. 2. Angle-resolved synchrotron radiated energy $\left(d \mathcal{E}_{\gamma} / d \theta_{\gamma}\right)$ in a uniform $\mathrm{C}^{6+}$ plasma slab with $n_{e}=17 n_{c}$, irradiated at a $10^{22} \mathrm{~W} \mathrm{~cm}^{-2}$ laser intensity. Three cases are considered: a semiinfinite laser wave in a semi-infinite plasma (blue), a 30-fs laser pulse in a semi-infinite plasma (green), and a 30 -fs laser pulse in a $1-\mu \mathrm{m}$ plasma (red). Angles are defined as $\theta_{\gamma}=\arccos \left(k_{\gamma, x} / k_{\gamma}\right) \in(0, \pi)$ ( $\mathbf{k}_{\gamma}$ is the photon wave vector) and are symmetrized relative to $\theta_{\gamma}=$ 0 . The laser-to-photon energy conversion efficiency, $\eta_{\gamma} \equiv \mathcal{E}_{\gamma} / \mathcal{E}_{L}$, is indicated in each case.

plasma. In the present case, however, synchrotron radiation causes dissipation, and more importantly, the laser profile is subject to transverse modulations (not shown), leading to local field maxima $\left|B_{z}\right| / B_{0} \approx 120$, so that the transverse canonical momentum is no longer conserved.

Interestingly, the $p_{x}$ profile of the return current presents anharmonic oscillations at $\lambda_{\text {mod }} \approx 1.5 \lambda_{L}$, resulting in strong density modulations $\left(\Delta n_{e} / n_{e} \gtrsim 1\right)$ inside the laser pulse. The related maxima in $\left|p_{x}\right|$, when coinciding with $B_{z}$ extrema, yield peaks in the radiated power density profile (translating into $\sim 5$-fs-time-scale fluctuations in the spatially averaged radiated power, not shown here). This is expected, as the high-energy counterstreaming electrons are those optimizing the quantum parameter $\chi_{e} \simeq \gamma\left(1-v_{x} / c\right) a_{L} \hbar \omega_{L} / m_{e} c^{2} \simeq$ $2 \gamma a_{L} \hbar \omega_{L} / m_{e} c^{2}$ (for purely counterstreaming electrons of typical energy $\gamma$ and longitudinal velocity $v_{x}$ ), resulting in a backward-directed radiated power (per electron), $P_{\mathrm{cl}} \simeq$ $(2 / 3) \alpha_{f} m_{e} c^{2} \chi_{e}^{2} / \tau_{C} \simeq(8 / 3)\left(r_{e} \omega_{L} / c\right) \omega_{L} m_{e} c^{2}\left(\gamma a_{L}\right)^{2}$, with $r_{e}$ being the classical electron radius and assuming negligible quantum corrections [49]. The large $\left|p_{y}\right|$ momenta of the counterstreaming electrons at the emission peaks account for the extended backward-directed emission lobe shown in the angular spectrum plotted (as the blue curve) in Fig. 2. A weaker and narrower forward-directed component is also visible, due to the reflected part of the laser wave being scattered by the forward-moving electrons. The total laser-to-photon energy conversion, defined as the fraction of the injected laser energy radiated into $\geqslant 10-\mathrm{keV}$-energy photons, is measured to be $\eta_{\gamma} \simeq 13 \%$ at the end of the simulation ( $t=150 \mathrm{fs}$ ).

The above emission scenario, hinging on the electrons injected back into the electromagnetic wave at the laser front, was first investigated in Refs. [69] and [87], where it was termed reinjected electron synchrotron emission, and found to yield the largest radiation yield at $I_{L} \gtrsim 10^{22} \mathrm{~W} \mathrm{~cm}^{-2}$. The overall description provided in those works is consistent with our results, except regarding the quantitative estimate of the radiation burst time scale ( $\sim 5$ fs here). This time was in- terpreted as that needed for the compressed electrons at the laser front to build up an electrostatic field $\left(E_{x} \simeq e n_{e 0} c t / \epsilon_{0}\right)$ exceeding the $\mathbf{v} \times \mathbf{B} \propto a_{L}$ force, thus reflecting them toward the laser source. This reasoning yields a 'breakdown time' [69], $\tau_{\mathrm{bd}} \simeq a_{L}\left(n_{c} / n_{e 0}\right) \omega_{L}^{-1}$. Under the present conditions, we should have $c \tau_{\text {bd }} \simeq 0.8 \mu \mathrm{m}$, which is about half the observed spacing of the $P_{\gamma}$ peaks, $\lambda_{\text {mod }}=1.5 \mu \mathrm{m}$.

Rather, we propose the following simple explanation for the modulations affecting the $p_{x}<0$ hot electrons. Let us consider their motion in the rest frame of the laser front, in which the Doppler-shifted laser frequency is $\omega_{L}^{\prime}=$ $\omega_{L} \sqrt{\left(1-v_{f} / c\right) /\left(1+v_{f} / c\right)}$ (assuming $\left.k_{L} \approx \omega_{L} / c\right)$. The electrons impinging on the laser front from the unperturbed plasma experience the $2 \omega_{L}^{\prime}$-oscillating component of the laser's ponderomotive force while being injected downstream at $v_{x} \approx-c$. As a consequence, a current modulation is induced with wave number $k_{\text {mod }}^{\prime}=-2 \omega_{L}^{\prime} / c$. In the laboratory frame, this wave number becomes $k_{\bmod }=-2 \gamma_{f}(1-$ $\left.v_{f} / c\right) \omega_{L}^{\prime} / c=-2\left[\left(1-v_{f} / c\right) /\left(1+v_{f} / c\right)\right] \omega_{L} / c$, corresponding to a wavelength

$$
\lambda_{\text {mod }}=\left[\left(1+v_{f} / c\right) /\left(1-v_{f} / c\right)\right] \lambda_{L} / 2 .
$$

In the present case, where $v_{f} / c \simeq 0.47$, one expects $\lambda_{\text {mod }} \approx$ $1.4 \mu \mathrm{m}$, in good agreement with the simulation.

The observation that the radiation is mainly backward directed and emitted as bursts throughout the irradiated region allows for a rough estimate of the total radiation yield,

$$
\eta_{\gamma}=\xi \frac{P_{\mathrm{cl}} n_{h<} v_{f} t}{I_{L}},
$$

where we have introduced $n_{h<}$, the density of the counterstreaming $\left(p_{x}<0\right)$ electrons, and $\xi$, the ratio of the burst length to its spacing, $\lambda_{\text {mod }}$. Further assuming a mean electron energy $\langle\gamma\rangle \approx a_{L}$-fairly consistent with Fig. 1(b)_-gives

$$
\eta_{\gamma} \approx \kappa \xi \frac{n_{h<}}{n_{c}} \frac{v_{f} t \omega_{L}}{c} a_{L}^{2},
$$

with $\kappa \equiv(16 / 3)\left(r_{e} \omega_{L} / c\right) \simeq 9.44 \times 10^{-8}$. Taking $n_{h<}=n_{e 0} / 2$ and $\xi=0.1$ leads to $\eta_{\gamma} \approx 8 \%$ at $t=150 \mathrm{fs}$. This value is comparable with the simulation value $\eta_{\gamma} \simeq 13 \%$. The difference is attributed to uncertainties in the estimation of the electron parameters, to modulations in the laser field strength, and to the neglect of the forward-directed radiation (due to the forward-moving electrons interacting with the light reflected off the laser front).

For completeness, we have repeated the same simulation with a Gaussian laser pulse of 30-fs FWHM duration, impinging onto either a semi-infinite or a $1-\mu \mathrm{m}$-thick $\mathrm{C}^{6+}$ plasma of electron density $n_{e 0}=17 n_{c}$. As expected, the semi-infinite plasma yields a spatially averaged radiated power at a pulse maximum $\left(d \mathcal{E}_{\gamma} / d t \simeq 0.045 \mathrm{~J} \mathrm{fs}^{-1} \mu \mathrm{m}^{-1}\right)$ close to that measured at the same time with a constant laser drive. It also leads to a similar radiated angular spectrum (compare the blue and green curves in Fig. 2), although with a more pronounced transverse component $\left(\theta_{\gamma} \simeq \pi / 2\right)$. This change is ascribed to the energy depletion of the short laser pulse as it propagates through the plasma, which leads to near-transparency interaction conditions and favors transverse emission, as observed previously $[72,82]$. Our simulation also predicts that due to progressive depletion of the laser pulse, the total radiated 

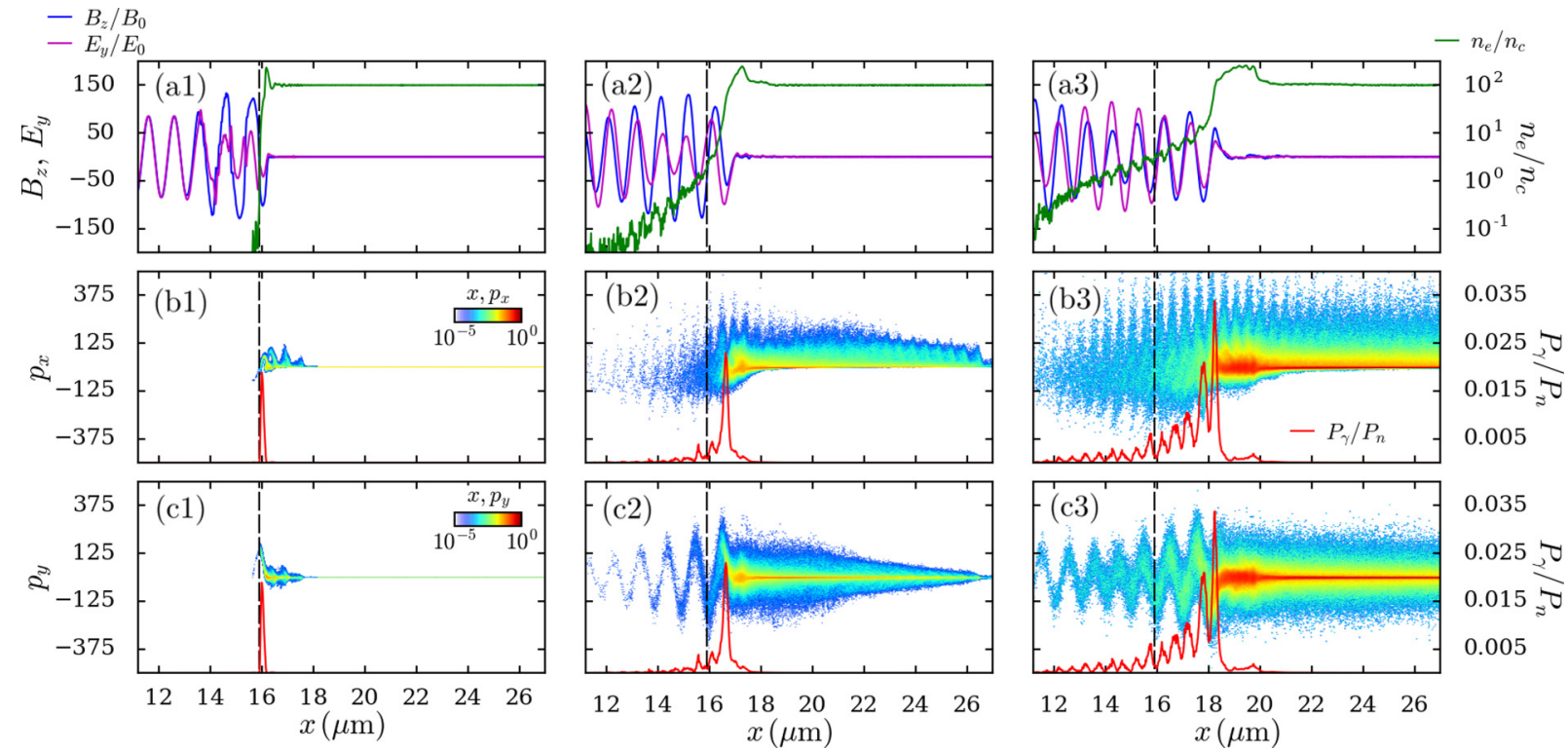

FIG. 3. Interaction of a semi-infinite, electromagnetic plane wave $\left(I_{L}=10^{22} \mathrm{~W} \mathrm{~cm}^{-2}\right)$ with a semi-infinite, relativistically overcritical $\mathrm{C}^{6+}$ plasma $\left(n_{e 0}=100 n_{c}\right)$. (a1-a3) Longitudinal lineouts of the $E_{y}$ (purple) and $B_{z}$ (blue) field components and of the electron density $n_{e}$ (green). (b1-b3) $x-p_{x}$ electron phase space (averaged along $y$ ) (red). (c1-c3) $x$ - $p_{y}$ electron phase space (averaged along $\left.y\right)$. In (b1)-(b3) and (c1)-(c3), the red curve plots a lineout of the synchrotron radiated power density, $P_{\gamma}$ (red). The three columns correspond to the interaction times: (a1-c1) $t=4 \mathrm{fs}$, (a2-c2) $36 \mathrm{fs}$, and (a3-c3) $100 \mathrm{fs}$.

power starts diminishing after $t \simeq 50 \mathrm{fs}$ and falls below $10 \%$ of its maximum value at $t \gtrsim 100 \mathrm{fs}$ (not shown).

When considering a finite $(1-\mu \mathrm{m})$ plasma thickness, the radiated power is reduced by approximately an order of magnitude $\left(\eta_{\gamma} \simeq 1 \%\right)$, and the emission is more concentrated in the backward direction (Fig. 2). There are two main reasons for these features. The first is that, unlike what occurs in a semi-infinite plasma, where the counterstreaming electrons that mainly account for high-energy radiation are continually replenished at the laser front (as long as the laser has not been strongly depleted), these are now electrostatically confined around the target, so that the radiation only occurs during the transit time of the laser pulse. Second, because of the short interaction time and the rapid plasma expansion, there is no significant laser reflection; this reduces the radiative contribution of the high-energy $p_{x}>0$ electrons and explains the vanishing forward emission.

\section{Relativistically overcritical plasma}

We now address the case of a semi-infinite, relativistically overcritical plasma $\left(n_{e 0} / n_{c}=100\right)$ illuminated by a semi-infinite, $10^{22} \mathrm{~W} \mathrm{~cm}^{-2}$ intensity laser wave. Figures 3(a1)-3(c3) present the main features of the interaction at three successive times.

The front-side electrons are energized through vacuum $/ J \times$ $B$ heating [88-90], leading to periodic injection of fast electron bunches into the plasma at twice the laser frequency. The $x-p_{x}$ and $x-p_{y}$ electron phase spaces of Figs. 3(b1)-3(c1) capture the instant $(t=4 \mathrm{fs})$ when the skin-layer electrons accelerated by the $E_{y}$ [purple curve in Fig. 3(a1)] component of the standing wave set up in vacuum (near the plasma boundary) have acquired their maximum transverse momenta and are being rotated by the $B_{z}$ field [blue curve in Fig. 3(a1)] toward the plasma [89]. The radiated power density [red curve in Figs. 3(b1)-3(c1)] peaks just in front of the steep plasma boundary, where $B_{z}$ is at its highest, and the accelerated electrons are characterized by $p_{x} \approx 120 m_{e} c>\left|p_{y}\right| \approx 50 m_{e} c$. This gives rise to a forward/oblique emission lobe extending from $\theta_{\gamma} \simeq 30^{\circ}$ to $\theta_{\gamma} \simeq 90^{\circ}$, as shown in the angular spectrum in Fig. 4 (blue curve). Note that the distorted $E_{y}$ and $B_{z}$ field profiles in vacuum [Fig. 3(a1)] are due to high-order harmonic generation from the oscillating plasma surface [91].

At a later time $(t=36 \mathrm{fs})$, the plasma temperature has strongly increased, and the plasma boundary, pushed by the laser's radiation pressure, has developed both a bump and a longer scale-length density profile [see Fig. 3(a2)]. The density bump is the signature of an electrostatic shock [92], which traps part of the fast electrons behind the laser "piston" (see the electrons with $p_{x}<0$ around $x \simeq 17-18 \mu \mathrm{m}$ ). The expanding dilute portion of the density profile $(x<16 \mu \mathrm{m})$ corresponds to the few electrons leaked through the ponderomotive barrier at the plasma boundary and moving across the standing wave. Synchrotron radiation then mainly occurs

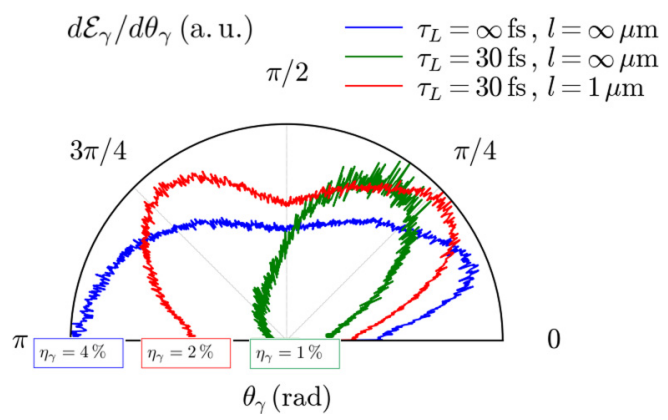

FIG. 4. Same as Fig. 2 for a $C^{6+}$ plasma with $n_{e}=100 n_{c}$. 
within an enlarged ( $\sim 0.3 \lambda_{L}$-thick) region that encompasses the skin layer and the lower-density electron cloud in front of it. The radiated power density culminates around electron densities of $\sim 20-40 n_{c}$, where the electron phase space shows high positive and negative $p_{x}$ values with, typically, $\left|p_{x}\right| \sim$ $p_{y}$. The resulting synchrotron emission is thus spread over a broad angular range in both the forward and the backward directions.

At an even later stage ( $t=100 \mathrm{fs}$ ), a larger number of electrons have escaped into the vacuum, forming an extended, relativistically undercritical shelf modulated at $\lambda_{L} / 2$ [Fig. 3(a3)]. There, the high-energy electrons exhibit an approximately even $p_{x}$ momentum distribution and mainly radiate around the $E_{y}$ (or $B_{z}$ ) extrema of the laser wave. Due to laser absorption, the radiation then mostly arises from the electrons counterstreaming against the incoming laser wave, hence accounting for the backward-directed $\left(\theta_{\gamma} \simeq \pi\right)$ lobe visible in Fig. 4. At the end of the simulation $(t=150 \mathrm{fs})$, about $4 \%$ of the incident light energy is radiated away, which is about three times less than at $n_{e 0}=17 n_{c}$.

If the plasma is irradiated by a 30 -fs laser pulse, the synchrotron efficiency drops to $\eta_{\gamma} \simeq 1 \%$, and due to the shortened interaction time, the above-discussed, late-time backward components of the synchrotron emission vanish (green curve in Fig. 4). Changing to a 1- $\mu$ m foil target (while keeping the same laser parameters) improves the radiation efficiency $\left(\eta_{\gamma} \simeq 2 \%\right)$, unlike in the $n_{e 0}=17 n_{c}$ case. This differing trend stems from the fact that in the latter transparent regime the radiation occurs volumetrically, and so the radiation yield decreases in thinner targets. At $n_{e 0}=100 n_{c}$, by contrast, the foil remains opaque throughout the interaction: the emission is confined to the front side (precisely, in the $\sim 5-$ $100 n_{c}$ density plasma shelf preceding the laser-compressed skin layer), and its efficiency increases when a larger number of high-energy electrons propagate against the laser wave, as happens due to electrostatic reflection at the target backside. Consequently, the radiation from the $1-\mu \mathrm{m}$ foil exhibits two forward- and backward-directed lobes, about symmetric relative to the transverse axis (red curve in Fig. 4).

\section{COMPETITION BETWEEN BREMSSTRAHLUNG AND SYNCHROTRON EMISSION IN COPPER FOIL TARGETS}

We now study the relative contributions of Bremsstrahlung and synchrotron emission to the total high-energy radiation from a laser-driven thin solid foil. In contrast to the few previous studies on this subject $[73,75,76]$, which essentially focused on the laser intensity dependence of those two radiative processes, we consider fixed laser parameters and a single target material $(\mathrm{Cu})$ and investigate, through $2 \mathrm{D}$ simulations, the influence of the target thickness, varied from a few nanometers to a few micrometers.

\section{A. Numerical setup and modeling}

The 2D simulations reported below consider a laser pulse propagating in the $+x$ direction, linearly polarized along $y$, with a wavelength $\lambda_{L}=1 \mu \mathrm{m}$ and a maximum intensity $I_{L}=10^{22} \mathrm{~W} \mathrm{~cm}^{-2}\left(a_{L}=85\right)$. Moreover, it has a Gaussian temporal profile of 50-fs FWHM and a Gaussian transverse profile of 5- $\mu \mathrm{m}$ FWHM. The target consists of a solid-density copper plasma slab of thickness $16 \mathrm{~nm} \leqslant l \leqslant 5 \mu \mathrm{m}$. It is initialized with a $200-\mathrm{eV}$ temperature and a $Z^{*}=25$ ionization state, corresponding to an electron density $n_{e 0} \simeq 2000 n_{c}$. Its front and rear sides are coated with 3.2-nm-thick hydrogen layers of atomic density $n_{\mathrm{H}}=50 n_{c}$, which model the hydrogen-rich surface contaminants usually responsible for proton beam generation in laser experiments [93]. Note that an ultrahigh-intensity contrast is implicitly assumed; otherwise, the front-side hydrogen layer is expected to be blown away by the laser prepulse.

The domain dimensions are $L_{x} \times L_{y}=127 \times 40 \mu \mathrm{m}^{2}$ with a mesh size $\Delta x=\Delta y=3.2 \mathrm{~nm}$. The number of macroparticles per cell and species is adjusted depending on the foil thickness to limit the numerical cost. Specifically, it is varied from 2000 to 375 for $l \in(16,32,51,100) \mathrm{nm}$ and from 40 to 10 for $l \in(0.5,1,5) \mu \mathrm{m}$. Absorbing boundary conditions for particles and fields are employed in both the $x$ and the $y$ directions. The simulations are run over durations ranging from $270 \mathrm{fs}(l=16 \mathrm{~nm})$ to $\approx 800 \mathrm{fs}(l=5 \mu \mathrm{m})$.

Besides Bremsstrahlung and synchrotron emission, all simulations self-consistently describe elastic Coulomb collisions as well as impact- and field-induced ionization [83,94]. Bremsstrahlung is modeled using the Monte Carlo scheme developed in Ref. [78], taking account of both Thomas-Fermi and Debye-type screening effects.

\section{B. Target thickness dependence of the radiation yield}

The energy conversion efficiencies $\left(\eta_{\gamma}\right)$ of Bremsstrahlung (cyan) and synchrotron emission (red) into $>10-\mathrm{keV}$ photons are plotted in Fig. 5(a) as a function of the target thickness. The synchrotron efficiency initially increases from $\eta_{\gamma}=0.8 \%$ at $l=16 \mathrm{~nm}$ to a maximum of $1.5 \%$ at $l=32 \mathrm{~nm}$. It slowly decreases at larger thicknesses, reaching $0.2 \%$ at $l=5 \mu \mathrm{m}$. By comparison, the Bremsstrahlung efficiency steadily rises with thicker targets, scaling as $\eta_{\gamma} \propto l^{1.5}$ in the thickness range considered. Specifically, it increases from $\eta_{\gamma}=2 \times 10^{-4} \%$ at $l=16 \mathrm{~nm}$ to $1 \%$ at $l=5 \mu \mathrm{m}$. An important finding is that the Bremsstrahlung and synchrotron curves cross each other for $l \simeq 1-2 \mu \mathrm{m}$, in which case they both attain $\eta_{\gamma} \simeq 0.3 \%$.

It is interesting to compare these results with the corresponding variations in the laser absorption and transmission rates, displayed in Fig. 5(b). The twofold increase in the absorption coefficient between $l=16 \mathrm{~nm}$ and $l=32 \mathrm{~nm}$ is similar to that observed in the synchrotron efficiency. The absorption culminates in a plateau around $l=32-50 \mathrm{~nm}$, which also encompasses the maxima of the synchrotron efficiency. The transmission coefficient reaches $\sim 56 \%$ at $l=16 \mathrm{~nm}$ and abruptly drops within the plateau (to $\sim 20 \%$ at $l=32 \mathrm{~nm}$ and $\sim 0.1 \%$ at $l=50 \mathrm{~nm}$ ). In light of the results in Sec. II, the slightly better synchrotron performance at $l=32 \mathrm{~nm}$ than at $l=50 \mathrm{~nm}$ is ascribed to the partial transparency of the target, which allows the electrons to experience the full strength of the laser fields.

The conditions of strong laser absorption and significant transmission that maximize synchrotron emission are also known to enhance ion acceleration from thin foils driven by femtosecond laser pulses [95-98]. The optimum thickness for ion acceleration has been found to be $l_{\text {ion }} \simeq 0.5 \lambda_{L} a_{L}\left(n_{c} / n_{e 0}\right)$ 

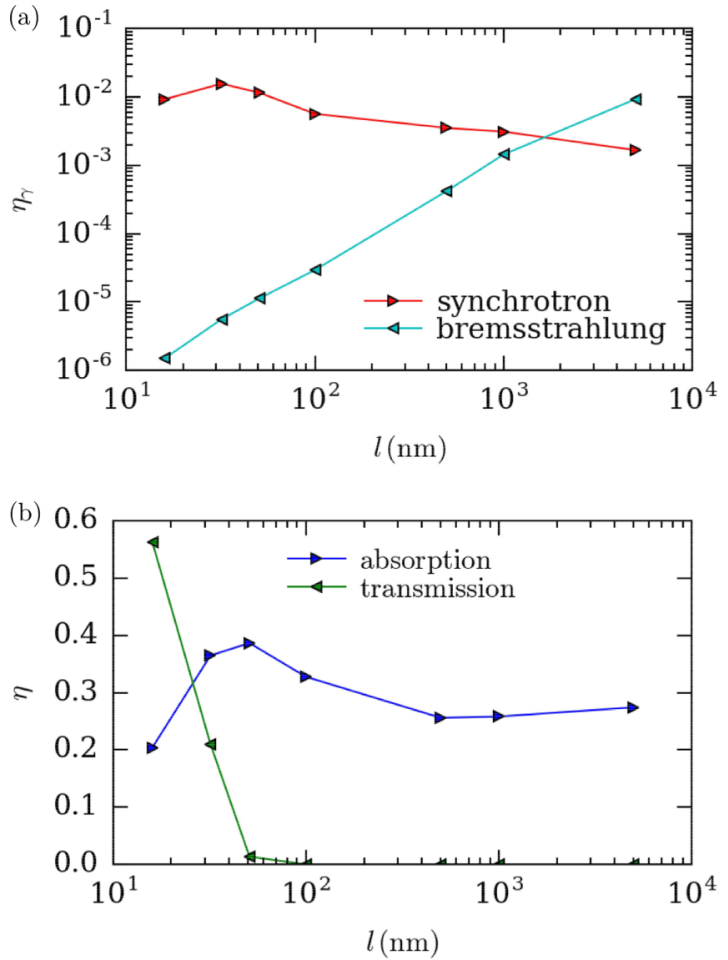

FIG. 5. Two-dimensional PIC simulations of an ultraintense $\left(10^{22} \mathrm{~W} \mathrm{~cm}^{-2}\right)$, ultrashort (50-fs), and tightly focused $(5-\mu \mathrm{m})$ laser pulse interacting with a copper foil target. (a) Synchrotron (red triangles) and Bremsstrahlung (cyan triangles) conversion efficiencies into $>10-\mathrm{keV}$ photons as a function of the target thickness. (b) Laser absorption (blue triangles) and transmission (green triangles) as a function of the target thickness.

$[96,97]$, close to the threshold thickness for self-induced relativistic transparency [99]. In the present case $\left(a_{L}=85\right.$, $n_{e 0} / n_{c}=2000$ ), one has $l_{\text {ion }} \simeq 21 \mathrm{~nm}$, a bit lower than the synchrotron-optimizing thickness $l \simeq 32 \mathrm{~nm}$.

At larger thicknesses $(l>50 \mathrm{~nm})$, our simulations predict that the absorption coefficient first decreases before stagnating at $\sim 25 \%$ for $l \geqslant 0.5 \mu \mathrm{m}$. This mere $\sim 30 \%$ decrease in the laser absorption is accompanied by a more pronounced (by an order of magnitude) drop in the synchrotron efficiency. This further shows that the laser absorption is not the only figure of merit for ensuring strong synchrotron emission.

\section{Illustrative cases}

\section{Transparent 32-nm-thick target}

We now focus on the radiation dynamics in the $l=32 \mu \mathrm{m}$ foil that maximizes the synchrotron efficiency. Figure 6(a) displays the time evolution of the synchrotron angular spectrum. A transition is seen to occur around the on-target laser peak ( $t=0 \mathrm{fs}$ ), which also coincides with the onset of relativistic transparency. Before transparency occurs, the $\mathrm{Cu}$ bulk plasma is compressed by the radiation pressure and set into motion as a whole-a process known as light-sail-type acceleration [100]. Some of the fast electrons (accelerated up to $\left|p_{x}\right| \simeq 100 m_{e} c$ ) recirculating around the bulk plasma are capable of passing through the laser piston to form a relativistically under-/near-critical shelf in front of the com-
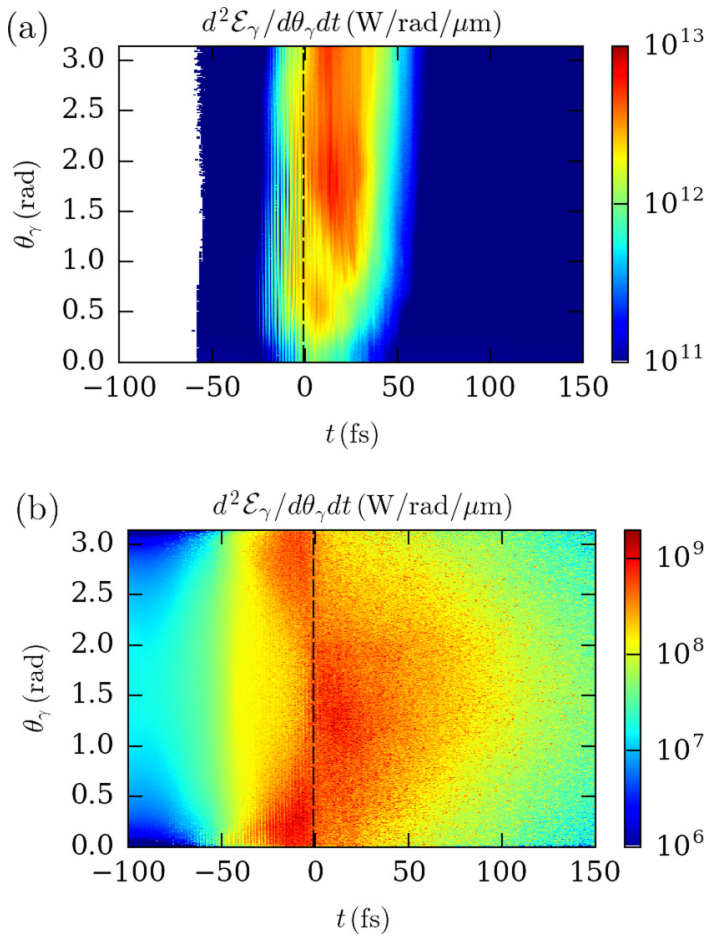

FIG. 6. Time evolution of the angle-resolved (a) synchrotron and (b) Bremsstrahlung power spectrum from a 32-nm-thick $\mathrm{Cu}$ foil target. The dashed line at $t \simeq 0 \mathrm{fs}$ indicates both the laser pulse maximum and the onset of relativistic transparency.

pressed boundary. As in the scenario considered in Sec. II C, synchrotron emission then mainly takes place in this relatively dilute $\left(10 n_{c}-100 n_{c}\right)$ expanding cloud. Due to significant laser reflection, both the forward- and the backward-moving highenergy electrons contribute to the radiation, the spectrum of which thus presents broad emission lobes in the forward and backward directions.

Figure 7 illustrates the laser-plasma interaction and the emissive region at $t=12 \mathrm{fs}$, just after the target has turned transparent to the laser light. This instant is when the

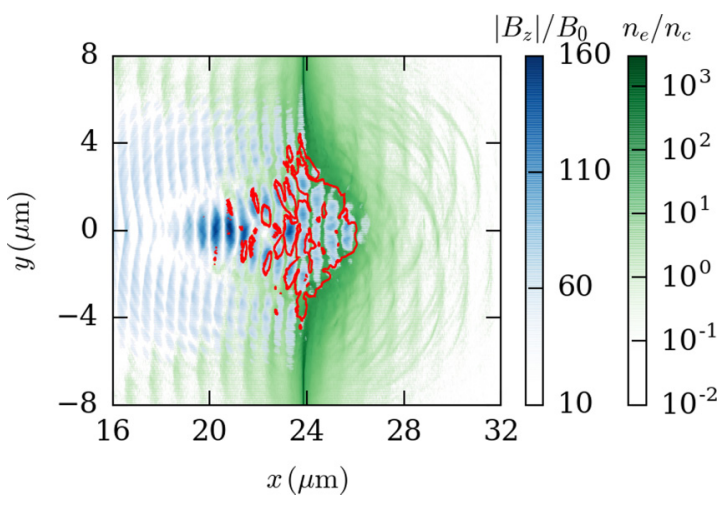

FIG. 7. A 32-nm-thick copper foil target: spatial distributions of the modulus of the magnetic field $\left|B_{z}\right|$ (blue color map) and the electron density $n_{e}$ (blue color map) at $t=12 \mathrm{fs}$ after the ontarget laser peak. The closed red curves are isocontour lines (at $P_{\gamma}=10^{23} \mathrm{~W} \mathrm{~cm}^{-3}$ ) of the synchrotron radiated power density. 
(spatially integrated) synchrotron power is at its highest. The pseudocolor maps show the spatial distributions of the absolute magnetic field strength (blue) and of the electron density (green). Overlaid is an isocountour (at $P_{\gamma}=$ $10^{23} \mathrm{~W} \mathrm{~cm}^{-3}$ ) of the instantaneous synchrotron power density (red). Rayleigh-Taylor-like modulations with spatial scale $\sim \lambda_{L}$ have developed in the irradiated region, breaking the translational invariance along $y$ and, hence, enhancing the electron energization (above $\left|p_{x}\right|=400 m_{e} c$ ) [89]. This disrupts the early-time balance between the radiation and the particle momentum fluxes [101], and leads to the accelerated $\mathrm{Cu}$ plasma being bored through by the laser pulse. Close inspection reveals that synchrotron emission is then concentrated in the laser-filled bulk plasma turned undercritical, of $\sim 3-\mu \mathrm{m}$ length and $3 n_{c}-40 n_{c}$ electron density. The time-resolved synchrotron energy spectrum, which was nearly isotropic early in the interaction, then increases in intensity and becomes mainly backward directed (at angles $\theta_{\gamma} \gtrsim \pi / 2$ ). As the laser pulse traverses the plasma, the average laser field strength experienced by the (electrostatically confined) relativistic electrons diminishes and so does the synchrotron power, which scales as $\sim\langle\gamma(t)\rangle a_{L}(t)^{2}$. The synchrotron emission becomes negligible once the laser pulse has traveled past the plasma ( $t \simeq 50 \mathrm{fs}$ ).

Figure 6(b) shows the Bremsstrahlung angular power spectrum as a function of time. Overall, its maximum values are about 4 orders of magnitude lower than those of the synchrotron spectrum [note the different scales of the color bars in Figs. 6(a) and 6(b)]. The Bremsstrahlung emission presents a strong rise at $t \simeq-50 \mathrm{fs}$ (when the on-target laser intensity reaches $\left.6.5 \times 10^{20} \mathrm{~W} \mathrm{~cm}^{-2}\right)$, in both the forward $\left(\theta_{\gamma}=\right.$ $0)$ and the backward $\left(\theta_{\gamma}=\pi\right)$ directions. This longitudinal emission is due to the high-energy electrons recirculating across the dense, high- $Z \mathrm{Cu}$ layer. At early times, the $\mathrm{Cu}$ layer is still planar and opaque to the laser field; therefore, owing to the quasi-1D interaction geometry, the transverse momenta of the fast electrons vanish inside the target, leading to peaked forward and backward emission lobes (recall that the Bremsstrahlung photons are emitted within an angular cone of $\sim \gamma^{-1}$ aperture along the electron direction). At later times, however, the angular distribution of the hot electrons broadens as a result of transverse surface modulations, and hence the angular Bremsstrahlung spectrum becomes increasingly isotropic.

Similarly to synchrotron radiation, the Bremsstrahlung radiated power culminates at the laser peak, yet decays away over a longer time scale ( $\sim 100$ vs $\sim 50$ fs for synchrotron) in the subsequent transparency regime. This decreasing trend can be understood from the following approximate expression of the (space-integrated) Bremsstrahlung power, valid in the ultrarelativistic limit and neglecting electron screening [102]:

$$
\begin{aligned}
\frac{d E_{b, h}}{d t}= & 12 \alpha r_{e}^{2} Z^{2} m_{e} c^{3} D_{h} l_{\mathrm{Cu}}\left\langle n_{\mathrm{Cu}}\right\rangle\left\langle n_{h}\right\rangle\left\langle\gamma_{h}\right\rangle \\
& \times\left[\log \left(2\left\langle\gamma_{h}\right\rangle\right)+0.92\right],
\end{aligned}
$$

where $l_{\mathrm{Cu}}$ denotes the longitudinal width of the expanding bulk copper plasma, with mean ion density $\left\langle n_{\mathrm{Cu}}\right\rangle$. We have also introduced $D_{h}$, the transverse width of the hotelectron cloud, with mean density $\left\langle n_{h}\right\rangle$ and energy $\left\langle\gamma_{h}\right\rangle$.
Note that the mean energy of Bremsstrahlung photons is $\langle\hbar \omega\rangle \approx m_{e} c^{2}\left\langle\gamma_{h}\right\rangle / 3$ [103]. Since the areal density $\left\langle n_{\mathrm{Cu}}\right\rangle l_{\mathrm{Cu}}$ is approximately constant, the Bremsstrahlung power should vary as $d E_{b, h} / d t \propto D_{h}\left\langle n_{h}\right\rangle\left\langle\gamma_{h}\right\rangle(t)$. Now, as the target expands and becomes increasingly quasineutral, most of the hot electrons are confined within the $\mathrm{Cu}$ bulk plasma, so that their longitudinal extent approximately coincides with $l_{\mathrm{Cu}}$. Introducing the total hot-electron energy $E_{h}(t) \simeq l_{\mathrm{Cu}} D_{h}\left\langle n_{h}\right\rangle\left\langle\gamma_{h}\right\rangle(t)$ and noting that $D_{h} l_{\mathrm{Cu}}\left\langle n_{h}\right\rangle \simeq \mathrm{cst}$, one obtains $d E_{b, h} / d t \propto$ $E_{h}(t) / l_{\mathrm{Cu}}(t)$.

By looking at the dynamics of the target expansion and of the particle kinetic energies, we have checked that the above scaling is consistent with the observed evolution of the Bremsstrahlung power following the laser pulse maximum (and the onset of the target transparency). Over the time span $0<t<100 \mathrm{fs}$, the spatial extent of the bulk $\mathrm{Cu}$ plasma varies by a factor of $\sim 1.5$, while owing to energy transfer to $\mathrm{Cu}$ ions (which carry about $30 \%$ of the laser energy at $t=100 \mathrm{fs}$ ), the total electron energy drops by a factor of $\sim 5$, so that $d E_{b, h} / d t$ should decay by a factor of $\sim 7.5$. This prediction reasonably agrees with the then measured fivefold decrease in the Bremsstrahlung power [calculated from integration over $\theta_{\gamma}$ of the spectrum shown Fig. 6(b)], which goes from $1.6 \times 10^{9}$ to

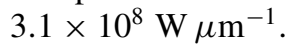

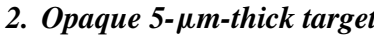

We now consider the radiation from a $5-\mu \mathrm{m} \mathrm{Cu}$ foil, that is, the thickest target considered in our 2D simulation study. This target remains opaque to the laser light throughout the interaction. At the final simulation time $(t=250 \mathrm{fs})$, while expanding at its rear and - to a lower extent - front sides, the thickness of the solid-density $\mathrm{Cu}$ layer is still about 4/5 of its initial value.

The time-resolved synchrotron angular power spectrum is displayed in Fig. 8(a). The synchrotron emission is observed to peak at $t \simeq 7 \mathrm{fs,} \mathrm{i.e.,} \mathrm{just} \mathrm{after} \mathrm{the} \mathrm{on-target} \mathrm{laser} \mathrm{maximum.}$ The corresponding spatial distributions of the laser field and electron density are shown in Fig. 9. Because of the sustained compression of the irradiated boundary, the electron density profile is locally much steeper than at the same time in the fast-expanding $l=32 \mathrm{~nm}$ foil (see Fig. 7), which, in turn, leads to significantly less energetic electrons (with longitudinal momenta up to $p_{x} \simeq 150 m_{e} c$ vs $p_{x}>400 m_{e} c$ at $l=$ $32 \mathrm{~nm}$ ). Such interaction conditions are close to those characterizing the early stage in Fig. 3. Accordingly, synchrotron emission arises in front of the laser-compressed boundary (see red isocontour at $P_{\gamma}=10^{23} \mathrm{~W} \mathrm{~cm}^{-3}$ in Fig. 7), where $10 \lesssim n_{e} \lesssim 100 n_{c}$, and is mainly forward directed $\left(\theta_{\gamma} \lesssim 1.5\right)$. Some backward emission also occurs by $t \simeq 20 \mathrm{fs}$, i.e., after a two-way transit time in the foil of the energetic electrons generated in the laser's rising edge, but contributes weakly to the total angular spectrum [see Figs. 10(a) and 12(b), discussed below].

Let us now examine the Bremsstrahlung spectrum presented in Fig. 8(b). Similarly to the $l=32 \mathrm{~nm}$ target, but to a greater extent given the two orders of magnitude larger thickness, a significant $\left(\sim 10^{10} \mathrm{~W} / \mathrm{rad} / \mu \mathrm{m}\right)$ isotropic background is radiated early on by the thermal electrons. The total Bremsstrahlung power increases by a factor of $\sim 10$ during the 

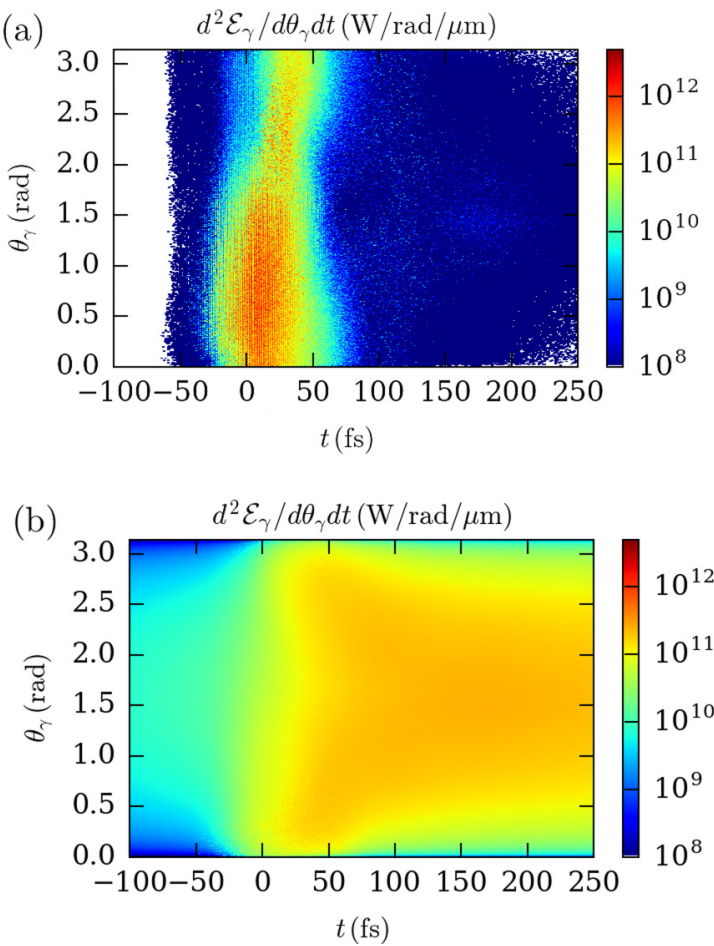

FIG. 8. Same as Fig. 6, from a 5- $\mu$ m-thick copper foil target.

main part of the pulse ( $-50 \mathrm{fs} \lesssim t \lesssim 50 \mathrm{fs}$ ) and essentially saturates afterwards. In such a thick target, the maximum simulation time $(t=+250 \mathrm{fs}$ after the on-target laser maximum) allowed by our computational resources is clearly too short for a quantitative evaluation of the total Bremsstrahlung yield. Except for this shortcoming, the Bremsstrahlung spectrum at $l=5 \mu \mathrm{m}$ evolves qualitatively as observed at $l=$ $32 \mathrm{~nm}$. Just after the laser peak, it is mainly contained in forward and backward lobes, and as time passes, it becomes increasingly isotropic due to the growing average isotropy of the electron distribution.

To clarify the electron relaxation dynamics, we have followed the time evolution of the longitudinal and transverse temperatures of two groups of electrons (the sum of which make up the whole electron population): those ('bulk') initially contained in the preionized $\mathrm{Cu}^{25+}$ layer and those

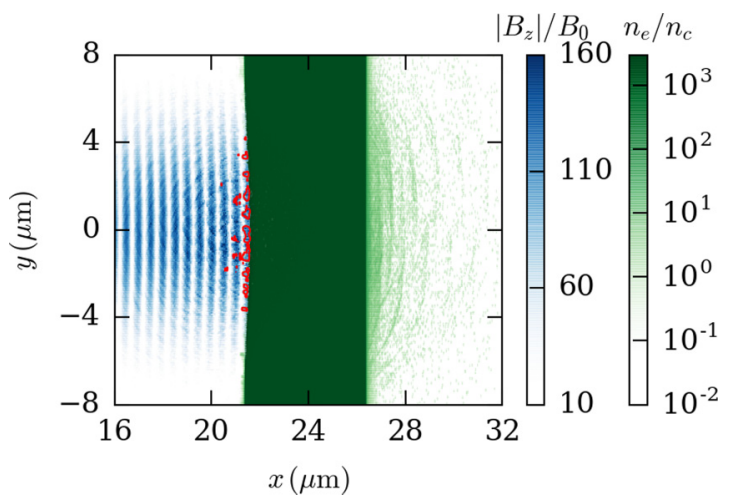

FIG. 9. Same as Fig. 7, from a 5- $\mu \mathrm{m}$-thick copper foil target.
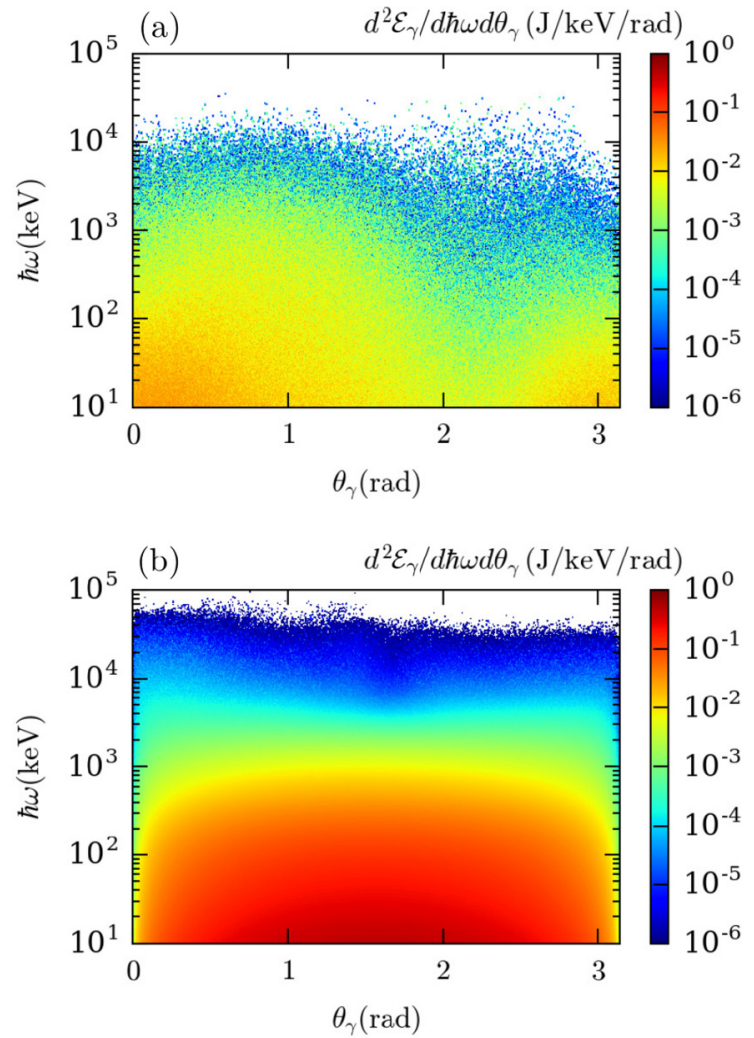

FIG. 10. Time-integrated (a) synchrotron and (b) Bremsstrahlung energy-angle spectra from a $5-\mu \mathrm{m}$-thick $\mathrm{Cu}$ target. The result is expressed as $\mathrm{J} / \mathrm{keV} / \mathrm{rad}$ instead of $\mathrm{J} / \mathrm{keV} / \mathrm{rad} / \mu \mathrm{m}$, as we assumed the third dimension $z$ to be $5 \mu \mathrm{m}$.

('ionized') issued from the surface hydrogen layers and subsequent ionization of the $\mathrm{Cu}$ ions. The latter group notably includes surface electrons directly laser-accelerated to high energies and, so, reaches much higher temperatures than the former group. The longitudinal $\left(T_{x}\right)$ and transverse $\left(T_{y}\right)$ temperatures of each group are defined as the momentum fluxes $T_{x, y}=\int d^{3} p f_{e}(\mathbf{p}) p_{x, y}^{2} / m_{e} \gamma\left(f_{e}\right.$ is the space-averaged electron momentum distribution). Figure 11(a) indicates that, as expected, the longitudinal temperature initially increases the most rapidly for both electron groups. Specifically, $T_{x \text {,ionized }}$ peaks (at $\sim 2 \mathrm{MeV}$ ) at the laser maximum, after which it steadily decreases down to $\sim 0.7 \mathrm{MeV}$ at $t=250 \mathrm{fs}$. Meanwhile, $T_{y, \text { ionized }}$, which is about two times lower at the laser maximum and continues rising up to $t \simeq 70 \mathrm{fs}$, at which time it overtakes $T_{x, \text { ionized }}$ before stagnating/slowly decreasing later on, so that $T_{y} / T_{x} \sim 1.4$ at the final time. This anisotropic relaxation is attributed to preferentially longitudinal momentum losses to the expanding ions and is more pronounced for the higher-energy electron fraction, as evidenced by the $p_{x}-p_{y}$ electron momentum distribution at $t=250 \mathrm{fs}$ [Fig. 11(b)]. Meanwhile, the 'bulk' electrons reach their maximal longitudinal $\left(T_{x, \text { bulk }} \simeq 50 \mathrm{keV}\right)$ and transverse $\left(T_{y, \text { bulk }} \simeq 30 \mathrm{keV}\right)$ values around $t \simeq 25 \mathrm{fs}$ and $t \simeq 70 \mathrm{fs}$, respectively. Due to collisional scattering off $\mathrm{Cu}$ ions, isotropization is reached at $t \simeq 90 \mathrm{fs}$ and is maintained throughout the subsequent cooling of the bulk electrons. 

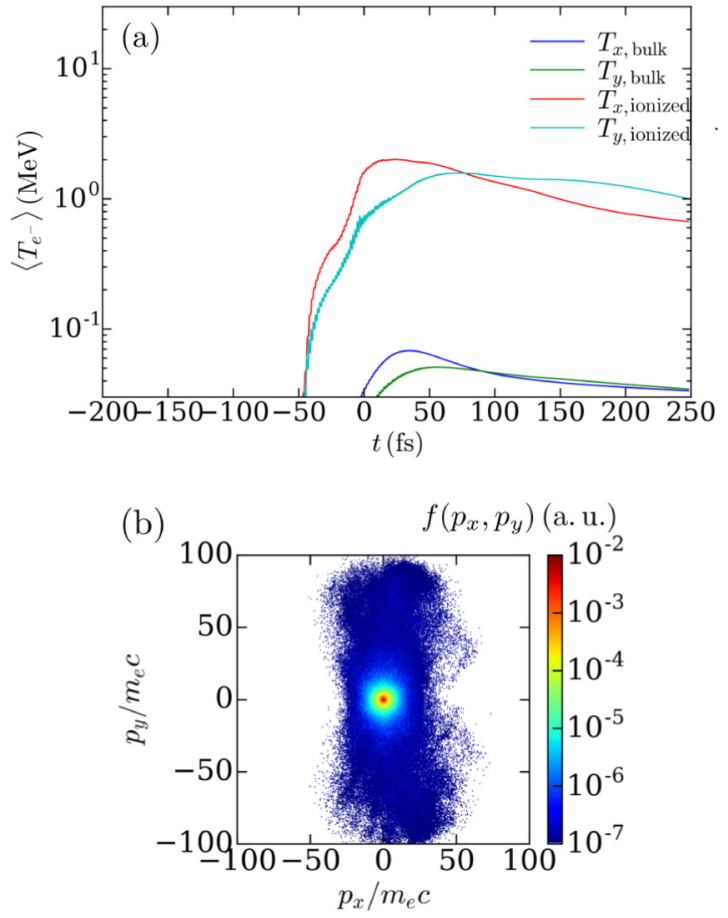

FIG. 11. (a) Time evolution of the longitudinal $\left(T_{x}\right)$ and transverse $\left(T_{y}\right)$ electron temperatures in a 5- $\mu$ m-thick $\mathrm{Cu}$ target. We distinguish between the ('bulk') electrons initially contained in the preionized $\mathrm{Cu}^{25+}$ layer and the ('ionized') electrons originating from the surface hydrogen layers and subsequent ionization of the $\mathrm{Cu}$ ions. (b) Space-integrated $p_{x}-p_{y}$ electron distribution at $t=+250 \mathrm{fs}$.

As a result, the time-integrated Bremsstrahlung energyangle spectrum plotted in Fig. 10(b) shows a nearly isotropic shape up to photon energies of $\sim 1 \mathrm{MeV}$. By contrast, the higher-energy photons, which are emitted by highly relativistic electrons, appear to be more collimated in the longitudinal (forward and backward) directions. Figure 8(b) indicates that this emission mainly takes place within $\sim 50$ fs after the laser maximum. Yet the $>1-\mathrm{MeV}$ energy photons carry only a very weak fraction $(\lesssim 1 \%)$ of the total Bremsstrahlung energy [Fig. 10(b)]. To conclude this part, we note that the late-time transverse anisotropy of the ultrarelativistic electrons [Fig. 11(b)] does not lead to a measurable signal because of their much reduced density fraction.

\section{Target thickness dependence of the radiation spectra}

The properties of the synchrotron and Bremsstrahlung emissions from copper foils of varying thicknesses are summarized in Figs. 12(a) and 12(b) and Figs. 13(a)-13(c).

The broad energy spectra of synchrotron radiation [Fig. 12(a)] exhibit similar monotonically decreasing shapes regardless of the target thickness. They confirm that the maximum yield is achieved at $l=32 \mathrm{~nm}$. The thicknesses $l=$ $32 \mathrm{~nm}$ and $51 \mathrm{~nm}$ produce the highest photon cutoff energies $(\sim 100 \mathrm{MeV})$, about twice as high as those obtained in micrometric $(l=1-5 \mu \mathrm{m})$ foils. The synchrotron angular spectra [Fig. 12(b)] evidence a clear transition between two distinct angular patterns when the target is made thicker: (i) A dominantly backward/transverse emission at $l=16-51 \mathrm{~nm}$, with
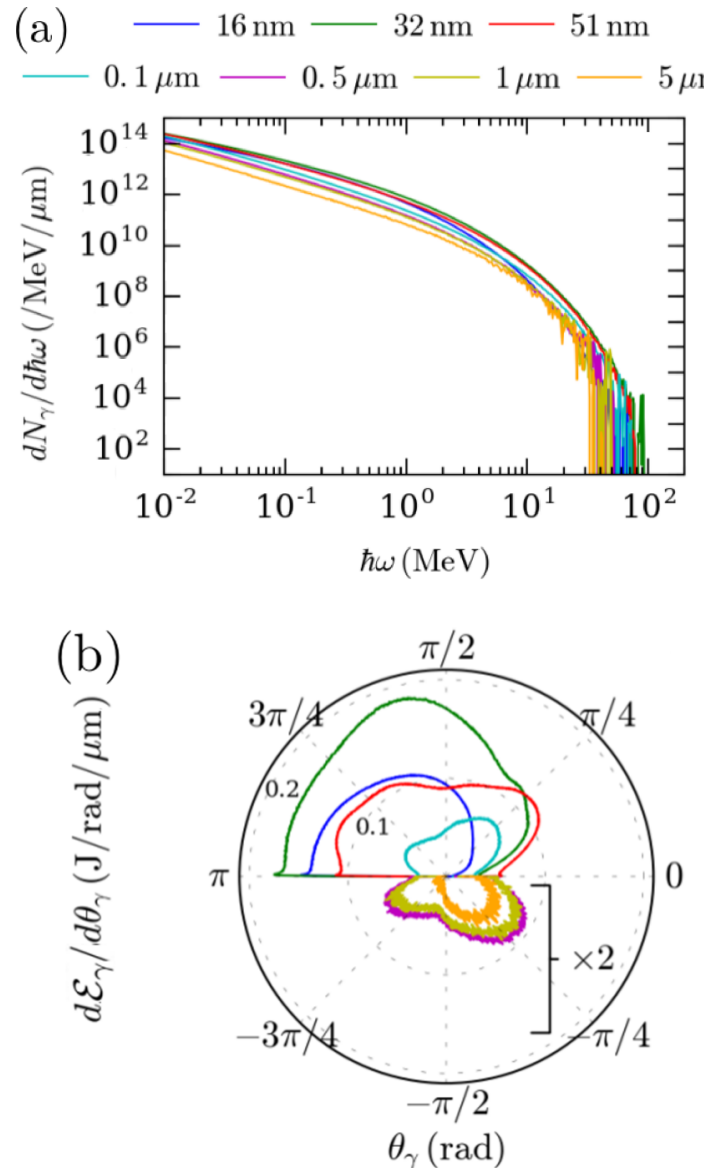

FIG. 12. Variations in the synchrotron radiation with the $\mathrm{Cu}$ target thickness: (a) Energy-resolved and (b) angle-resolved radiated energy spectra for $>10-\mathrm{keV}$ photon energies. In (b), the energy densities at thicknesses of $\leqslant 0.1 \mu \mathrm{m}$ (lower half-plane) have been multiplied by a factor of 2 for visibility.

an oblique forward lobe emerging at larger $l$; and (ii) a mainly oblique forward emission at $l=0.5-5 \mu \mathrm{m}$, with a weaker backward lobe, due to refluxing electrons and diminishing at larger $l$.

The Bremsstrahlung energy spectra [Fig. 13(a)] corroborate the growing trend of the Bremsstrahlung yield with the foil thickness as revealed in Fig. 5(a). As also expected from Fig. 5(a), they show stronger variations with $l$ than the synchrotron spectra, across the full range of photon energies. Moreover, they share roughly the same photon cutoff energy $(\sim 100 \mathrm{MeV})$, similar to that of synchrotron emission from nanometric foils.

The Bremsstrahlung angular spectra are displayed in Figs. 13(b) and 13(c) for two photon groups. The Bremsstrahlung photons with energies of $\hbar \omega \geqslant 10 \mathrm{keV}$ [Fig. 13(b)] are radiated at all angles, but their emission tends to be maximized in the forward direction for $l \leqslant 0.1 \mu \mathrm{m}$ (yet the Bremsstrahlung yield is then very weak) while it is essentially isotropic in $l \geqslant 0.5 \mu \mathrm{m}$ targets (note that an isotropic power spectrum scales with the polar angle as $\sin \theta_{\gamma}$, as observed at $l \geqslant 0.5 \mu \mathrm{m})$ due to the dominant contribution of the isotropized moderate-energy $(\lesssim 1-\mathrm{MeV})$ electrons. 
$-16 \mathrm{~nm}-32 \mathrm{~nm}-51 \mathrm{~nm}-0.1 \mu \mathrm{m}-0.5 \mu \mathrm{m}-1 \mu \mathrm{m}-5 \mu \mathrm{m}$

(a) $\forall \hbar \omega \geq 10 \mathrm{keV}$

(b) $\forall \hbar \omega \geq 10 \mathrm{keV}$

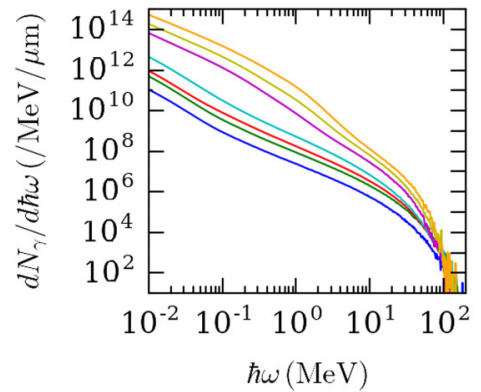

(c) $\forall \hbar \omega \geq 5 \mathrm{MeV}$

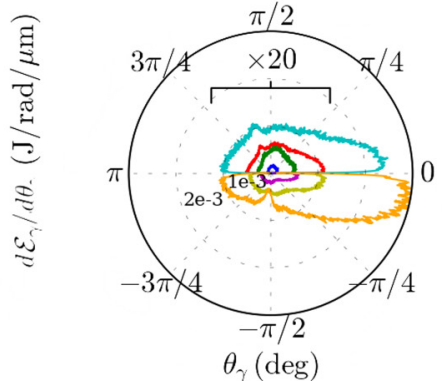

FIG. 13. Variations in the Bremsstrahlung radiation with the $\mathrm{Cu}$ target thickness. (a) Energy-resolved and (b, c) angle-resolved radiated energy spectra for (b) $>10-\mathrm{keV}$ and (c) $>5-\mathrm{MeV}$ photon energies. In (b) and (c), the energy densities at thicknesses of $\leqslant 0.1 \mu \mathrm{m}$ (upper half-planes) have been multiplied by factors of 200 and 20, respectively.

Photons with $\hbar \omega \geqslant 5 \mathrm{MeV}$, on the other hand, are increasingly collimated along the laser axis (and especially in the forward direction) at larger thicknesses [Fig. 13(c)]. The reason for this trend is that the ultrarelativistic electrons emitting those photons are generated preferentially along the laser axis (coinciding with the target normal) and can recirculate a few times across the solid target (hence the well-defined forward and backward lobes at $l=5 \mu \mathrm{m}$ ) before losing longitudinal momentum through ion expansion (slowed down at large $l$ ) or collisions.

\section{CONCLUSIONS}

Using advanced particle-in-cell simulations, we have numerically studied the processes of high-energy radiation in plasmas of various kinds, irradiated by laser waves of $10^{22} \mathrm{~W} \mathrm{~cm}^{-2}$ intensity. Following several previously published works, first, we have reexamined the energy and angular properties of synchrotron radiation in simplified interaction scenarios, involving laser plane waves of infinite or finite duration and fully ionized, uniform-density plasma slabs of semi-infinite or finite thickness. Our simulations confirm the existence of distinct synchrotron emission regimes depending on the density, and therefore the transparency or opacity, of the driven plasma.

At relativistically undercritical density $\left(n_{e}=17 n_{c}\right)$, the photon emission is mainly caused by energetic electrons counterstreaming against the laser wave. These electrons are injected at high energies toward the laser source across the laser front, in a time-modulated way due to relativistic Doppler effects. As a result, backward-directed radiation bursts are produced throughout the whole laser-filled volume. While forward emission is also significant in semi-infinite plasmas due to nonnegligible reflected light (interacting with forward-moving electrons), it is found to essentially vanish in rapidly expanding, $1-\mu \mathrm{m}$-thick targets.

At overcritical density $\left(n_{e}=100 n_{c}\right)$, photon emission initially occurs in the narrow vacuum region where the electrons are energized and, at later times, in a more extended region encompassing the skin layer and a fraction of the expanding preplasma. In semi-infinite opaque targets, the radiation is dominated by electrons being rotated back to the target and, thus, exhibits a broad maximum at forward angles (around $\theta_{\gamma} \simeq 1$ ). In $1-\mu$ m-thick targets, the radiation is enhanced with two forward and backward lobes owing to recirculating electrons.

Second, we have investigated the competition of the synchrotron and Bremsstrahlung emissions driven by a $10^{22} \mathrm{~W} \mathrm{~cm}^{-2}$ intensity, 50-fs laser pulse focused onto solid copper foils, with thicknesses ranging from a few tens of nanometers to a few micrometers. We have looked in great detail into the dynamics and spectral properties of both radiation processes and correlated them with the ultrafast evolution of the target. We have found that the synchrotron efficiency is maximized (reaching an $\sim 1 \%$ conversion efficiency into $>10-\mathrm{keV}$ photons) in $\sim 30$ - to 50 -nm-thick foils which, owing to relativistic and expansion effects, transition from being opaque to being transparent during the laser pulse. In this interaction regime, the synchrotron emission takes place throughout the expanding bulk plasma and is dominated by ultrarelativistic electrons counterpropagating against the incoming wave. The rapid drop in plasma densities then leads to very weak Bremsstrahlung radiation. As the target is made thicker and opaque to the laser pulse, both hot-electron generation and synchrotron emission get localized around the target front side. The synchrotron spectrum is then mainly forward directed yet may also feature a backward lobe due to electron recirculation during the laser irradiation. As the target expands more slowly with larger thickness, the energized electrons experience higher average densities, which enables efficient Bremsstrahlung over longer time scales. Bremsstrahlung exhibits stronger variations with the target thickness than synchrotron and turns out to be the dominant radiation source in $\mathrm{Cu}$ targets of $l \gtrsim 1 \mu \mathrm{m}$ thickness, with a conversion efficiency reaching the percentage level. While most of the Bremsstralhung energy into $\geqslant 10-\mathrm{keV}$ photons is then radiated isotropically due to the prevailing contribution of relatively low-energy isotropized electrons, its high-energy $(\geqslant 5-\mathrm{MeV})$ fraction is emitted within increasingly collimated forward and backward lobes.

Our numerical study has led to a better understanding of high-energy radiation in laser-plasma interactions at classically overcritical densities. In contrast to many previous works in this fast-developing research field, which have 
considered the effect of raising the laser intensity up to values $\left(I_{L} \sim 10^{23-24} \mathrm{~W} \mathrm{~cm}^{-2}\right)$ beyond the reach of near-future experiments, we have considered a fixed on-target laser intensity of $I_{L} \sim 10^{22} \mathrm{~W} \mathrm{~cm}^{-2}$, soon achievable experimentally, and examined, within a high-resolution, self-consistent simulation framework, the influence of the thickness of a simple foil target in a previously unexplored parameter range. In so doing, we have highlighted the as-yet-overlooked impact of the ultrafast target expansion on high-energy radiation. Using this readily realizable setup, we have identified an optimal range of (nanometric) target thicknesses for dominant synchrotron emission, associated with an interaction effectively taking place in the relativistic transparency regime, that is, without the need for sophisticated targetry such as cryogenic hydrogen targets [104] or additional laser pulses for plasma conditioning. We have also evidenced a characteristic evolution (i.e., from mainly backward to mainly forward) of the angular synchrotron spectrum as the target is made thicker and, hence, transitions from being effectively transparent to being opaque.

Our results therefore pave the way for the exploration of high-efficiency $(\sim 1 \%)$, synchrotron photon sources from laser-solid interactions at the forthcoming ELI-class facilities, assuming that these feature a temporal contrast high enough to allow nanometer-scale foil targets to be used. Our study has also revealed how the size of the copper foil targets, and their resulting expansion, critically determines the yield and energy-angle distribution of Bremsstrahlung and that the latter mechanism provides the main channel for high-energy radiation in copper foils a few micrometers thick. Extending this simulation work to a broader range of laser (duration, intensity, focal spot) and target (material, geometry) parameters and, in the longer term, to a real-world 3D geometry, leaves room for further improvement.

To conclude, we believe that, besides clearing up the interplay of plasma and radiation processes in ultrafast, ultrarelativistic laser-solid interactions, and thus being of fundamental interest to a growing research community, this work will be most useful to guide and interpret related near-future experiments.

\section{ACKNOWLEDGMENTS}

E.d.H. was supported by French National Research Agency project TULIMA (ANR-17-CE30-0033-01) and U.S. Air Force project AFOSR No. FA9550-17-1-0382. The authors acknowledge GENCI, France, for awarding us access to HPC resources at TGCC/CCRT (Allocation No. A0010506129).
[1] S. C. Wilks, W. L. Kruer, M. Tabak, and A. B. Langdon, Absorption of Ultra-Intense Laser Pulses, Phys. Rev. Lett. 69, 1383 (1992).

[2] E. Lefebvre and G. Bonnaud, Nonlinear electron heating in ultrahigh-intensity-laser-plasma interaction, Phys. Rev. E 55, 1011 (1997).

[3] J. R. Davies, Laser absorption by overdense plasmas in the relativistic regime, Plasma Phys. Control. Fusion 51, 014006 (2009).

[4] A. J. Kemp, F. Fiuza, A. Debayle, T. Johzaki, W. B. Mori, P. K. Patel, Y. Sentoku, and L. O. Silva, Laser-plasma interactions for fast ignition, Nucl. Fusion 54, 054002 (2014).

[5] J. D. Kmetec, C. L. Gordon, J. J. Macklin, B. E. Lemoff, G. S. Brown, and S. E. Harris, MeV X-Ray Generation with a Femtosecond Laser, Phys. Rev. Lett. 68, 1527 (1992).

[6] C. Gahn, G. Pretzler, A. Saemann, G. D. Tsakiris, K. J. Witte, D. Gassmann, T. Schatz, U. Schramm, P. Thirolf, and D. Habs, $\mathrm{MeV} \gamma$-ray yield from solid targets irradiated with fs-laser pulses, Appl. Phys. Lett. 73, 3662 (1998).

[7] M. D. Perry, J. A. Sefcik, T. Cowan, S. Hatchett, A. Hunt, M. Moran, D. Pennington, R. Snavely, and S. C. Wilks, Hard $\mathrm{X}$-ray production from high intensity laser solid interactions (invited), Rev. Sci. Instrum. 70, 265 (1999).

[8] S. P. Hatchett, C. G. Brown, T. E. Cowan, E. A. Henry, J. S. Johnson, M. H. Key, J. A. Koch, A. B. Langdon, B. F. Lasinski, R. W. Lee, A. J. Mackinnon, D. M. Pennington, M. D. Perry, T. W. Phillips, M. Roth, T. C. Sangster, M. S. Singh, R. A. Snavely, M. A. Stoyer, S. C. Wilks, and K. Yasuike, Electron, photon, and ion beams from the relativistic interaction of Petawatt laser pulses with solid targets, Phys. Plasmas 7, 2076 (2000).
[9] R. D. Edwards, M. A. Sinclair, T. J. Goldsack, K. Krushelnick, F. N. Beg, E. L. Clark, A. E. Dangor, Z. Najmudin, M. Tatarakis, B. Walton, M. Zepf, K. W. D. Ledingham, I. Spencer, P. A. Norreys, R. J. Clarke, R. Kodama, Y. Toyama, and $\mathrm{M}$. Tampo, Characterization of a gamma-ray source based on a laser-plasma accelerator with applications to radiography, Appl. Phys. Lett. 80, 2129 (2002).

[10] J. Galy, M. Mau, D. J. Hamilton, R. Edwards, and J. Magill, Bremsstrahlung production with high-intensity laser matter interactions and applications, New J. Phys. 9, 23 (2007).

[11] A. Compant La Fontaine, C. Courtois, and E. Lefebvre, Production of multi-MeV Bremsstrahlung x-ray sources by petawatt laser pulses on various targets, Phys. Plasmas 19, 023104 (2012).

[12] K. Yasuike, M. H. Key, S. P. Hatchett, R. A. Snavely, and K. B. Wharton, Hot electron diagnostic in a solid laser target by $\mathrm{K}$-shell lines measurement from ultraintense laser-plasma interactions $\left(3 \times 10^{20} \mathrm{~W} / \mathrm{cm}^{2}, \leqslant 400 \mathrm{~J}\right)$, Rev. Sci. Instrum. 72, 1236 (2001).

[13] R. B. Stephens, R. A. Snavely, Y. Aglitskiy, F. Amiranoff, C. Andersen, D. Batani, S. D. Baton, T. Cowan, R. R. Freeman, T. Hall, S. P. Hatchett, J. M. Hill, M. H. Key, J. A. King, J. A. Koch, M. Koenig, A. J. MacKinnon, K. L. Lancaster, E. Martinolli, P. Norreys, E. Perelli-Cippo, M. Rabec Le Gloahec, C. Rousseaux, J. J. Santos, and F. Scianitti, $\mathrm{K}_{\alpha}$ fluorescence measurement of relativistic electron transport in the context of fast ignition, Phys. Rev. E 69, 066414 (2004).

[14] A. B. Sefkow, G. R. Bennett, M. Geissel, M. Schollmeier, B. C. Franke, and B. W. Atherton, Efficiency Enhancement for $\mathrm{K}_{\alpha} \mathrm{X}$-Ray Yields from Laser-Driven Relativistic Electrons in Solids, Phys. Rev. Lett. 106, 235002 (2011). 
[15] Y. Glinec, J. Faure, L. Le Dain, S. Darbon, T. Hosokai, J. J. Santos, E. Lefebvre, J. P. Rousseau, F. Burgy, B. Mercier, and V. Malka, High-Resolution $\gamma$-Ray Radiography Produced by a Laser-Plasma Driven Electron Source, Phys. Rev. Lett. 94, 025003 (2005).

[16] A. Ravasio, M. Koenig, S. Le Pape, A. Benuzzi-Mounaix, H. S. Park, C. Cecchetti, P. Patel, A. Schiavi, N. Ozaki, A. Mackinnon, B. Loupias, D. Batani, T. Boehly, M. Borghesi, R. Dezulian, E. Henry, M. Notley, S. Bandyopadhyay, R. Clarke, and T. Vinci, Hard x-ray radiography for density measurement in shock compressed matter, Phys. Plasmas 15, 060701 (2008).

[17] H.-S. Park, B. R. Maddox, E. Giraldez, S. P. Hatchett, L. T. Hudson, N. Izumi, M. H. Key, S. Le Pape, A. J. MacKinnon, A. G. MacPhee, P. K. Patel, T. W. Phillips, B. A. Remington, J. F. Seely, R. Tommasini, R. Town, J. Workman, and E. Brambrink, High-resolution 17-75 keV backlighters for highenergy density experiments, Phys. Plasmas 15, 072705 (2008).

[18] E. Brambrink, H. G. Wei, B. Barbrel, P. Audebert, A. BenuzziMounaix, T. Boehly, T. Endo, C. D. Gregory, T. Kimura, R. Kodama, N. Ozaki, H.-S. Park, and M. Koenig, Direct density measurement of shock-compressed iron using hard $\mathrm{x}$ rays generated by a short laser pulse, Phys. Rev. E 80, 056407 (2009).

[19] B. Westover, A. MacPhee, C. Chen, D. Hey, T. Ma, B. Maddox, H.-S. Park, B. Remington, and F. N. Beg, Study of silver $K_{\alpha}$ and bremsstrahlung radiation from short-pulse lasermatter interactions with applications for x-ray radiography, Phys. Plasmas 17, 082703 (2010).

[20] C. Courtois, R. Edwards, A. C. L. Fontaine, C. Aedy, M. Barbotin, S. Bazzoli, L. Biddle, D. Brebion, J. L. Bourgade, D. Drew, M. Fox, M. Gardner, J. Gazave, J. M. Lagrange, O. Landoas, L. L. Dain, E. Lefebvre, D. Mastrosimone, N. Pichoff, G. Pien, M. Ramsay, A. Simons, N. Sircombe, C. Stoeckl, and K. Thorp, High-resolution multi-mev X-ray radiography using relativistic laser-solid interaction, Phys. Plasmas 18, 023101 (2011).

[21] L. C. Jarrott, A. J. Kemp, L. Divol, D. Mariscal, B. Westover, C. McGuffey, F. N. Beg, M. Suggit, C. Chen, D. Hey, B. Maddox, J. Hawreliak, H.-S. Park, B. Remington, M. S. Wei, and A. MacPhee, $\mathrm{K}_{\alpha}$ and bremsstrahlung X-ray radiation backlighter sources from short pulse laser driven silver targets as a function of laser pre-pulse energy, Phys. Plasmas 21, 031211 (2014).

[22] L. Antonelli, S. Atzeni, A. Schiavi, S. D. Baton, E. Brambrink, M. Koenig, C. Rousseaux, M. Richetta, D. Batani, P. ForestierColleoni, E. Le Bel, Y. Maheut, T. Nguyen-Bui, X. Ribeyre, and J. Trela, Laser-driven shock waves studied by $\mathrm{X}$-ray radiography, Phys. Rev. E 95, 063205 (2017).

[23] P. Audebert, P. Renaudin, S. Bastiani-Ceccotti, J.-P. Geindre, C. Chenais-Popovics, S. Tzortzakis, V. Nagels-Silvert, R. Shepherd, I. Matsushima, S. Gary, F. Girard, O. Peyrusse, and J.-C. Gauthier, Picosecond Time-Resolved X-Ray Absorption Spectroscopy of Ultrafast Aluminum Plasmas, Phys. Rev. Lett. 94, 025004 (2005).

[24] L. Lecherbourg, P. Renaudin, S. Bastiani-Ceccotti, J.-P. Geindre, C. Blancard, P. Cossé, G. Faussurier, R. Shepherd, and P. Audebert, X-ray absorption of a warm dense aluminum plasma created by an ultra-short laser pulse, High Energy Density Phys. 3, 175 (2007).

[25] F. Pisani, A. Bernardinello, D. Batani, A. Antonicci, E. Martinolli, M. Koenig, L. Gremillet, F. Amiranoff, S. Baton, J.
Davies, T. Hall, D. Scott, P. Norreys, A. Djaoui, C. Rousseaux, P. Fews, H. Bandulet, and H. Pepin, Experimental evidence of electric inhibition in fast electron penetration and of electricfield-limited fast electron transport in dense matter, Phys. Rev. E 62, R5927(R) (2000).

[26] M. I. K. Santala, M. Zepf, I. Watts, F. N. Beg, E. Clark, M. Tatarakis, M. Zepf, K. Krushelnick, A. E. Dangor, T. McCanny, I. Spencer, R. P. Singhal, K. W. D. Ledingham, S. C. Wilks, A. C. Machacek, J. S. Wark, R. Allott, R. J. Clarke, and P. A. Norreys, Effect of the Plasma Density Scale Length on the Direction of Fast Electrons in Relativistic Laser-Solid Interactions, Phys. Rev. Lett. 84, 1459 (2000).

[27] E. Martinolli, M. Koenig, S. D. Baton, J. J. Santos, F. Amiranoff, D. Batani, E. Perelli-Cippo, F. Scianitti, L. Gremillet, R. Mélizzi, A. Decoster, C. Rousseaux, T. A. Hall, M. H. Key, R. Snavely, A. J. MacKinnon, R. R. Freeman, J. A. King, R. Stephens, D. Neely, and R. J. Clark, Fast-electron transport and heating of solid targets in high-intensity laser interactions measured by $K \alpha$ fluorescence, Phys. Rev. E 73, 046402 (2006).

[28] C. D. Chen, P. K. Patel, D. S. Hey, A. J. Mackinnon, M. H. Key, K. U. Akli, T. Bartal, F. N. Beg, S. Chawla, H. Chen, R. R. Freeman, D. P. Higginson, A. Link, T. Y. Ma, A. G. MacPhee, R. B. Stephens, L. D. Van Woerkom, B. Westover, and M. Porkolab, Bremsstrahlung and $\mathrm{K} \alpha$ fluorescence measurements for inferring conversion efficiencies into fast ignition relevant hot electrons, Phys. Plasmas 16, 082705 (2009).

[29] A. L. Meadowcroft and R. D. Edwards, High-energy bremsstrahlung diagnostics to characterize hot-electron production in short-pulse laser-plasma experiments, IEEE Trans. Plasma Sci. 40, 1992 (2012).

[30] C. Zulick, B. Hou, F. Dollar, A. Maksimchuk, J. Nees, A. G. R. Thomas, Z. Zhao, and K. Krushelnick, High resolution bremsstrahlung and fast electron characterization in ultrafast intense laser-solid interactions, New J. Phys. 15, 123038 (2013).

[31] T. E. Cowan, A. W. Hunt, T. W. Phillips, S. C. Wilks, M. D. Perry, C. Brown, W. Fountain, S. Hatchett, J. Johnson, M. H. Key, T. Parnell, D. M. Pennington, R. A. Snavely, and Y. Takahashi, Photonuclear Fission from High Energy Electrons from Ultraintense Laser-Solid Interactions, Phys. Rev. Lett. 84, 903 (2000).

[32] K. W. D. Ledingham, P. McKenna, and R. P. Singhal, Applications for nuclear phenomena generated by ultra-intense lasers, Science 300, 1107 (2003).

[33] H. Schwoerer, F. Ewald, R. Sauerbrey, J. Galy, J. Magill, V. Rondinella, R. Schenkel, and T. Butz, Fission of actinides using a tabletop laser, Europhys. Lett. 61, 47 (2003).

[34] I. Pomerantz, E. McCary, A. R. Meadows, A. Arefiev, A. C. Bernstein, C. Chester, J. Cortez, M. E. Donovan, G. Dyer, E. W. Gaul, D. Hamilton, D. Kuk, A. C. Lestrade, C. Wang, T. Ditmire, and B. M. Hegelich, Ultrashort Pulsed Neutron Source, Phys. Rev. Lett. 113, 184801 (2014).

[35] X. L. Wang, Z. Y. Xu, W. Luo, H. Y. Lu, Z. C. Zhu, and X. Q. Yan, Transmutation prospect of long-lived nuclear waste induced by high-charge electron beam from laser plasma accelerator, Phys. Plasmas 24, 093105 (2017).

[36] E. P. Liang, S. C. Wilks, and M. Tabak, Pair Production by Ultraintense Lasers, Phys. Rev. Lett. 81, 4887 (1998). 
[37] C. Gahn, G. D. Tsakiris, G. Pretzler, K. J. Witte, C. Delfin, C.-G. Wahlström, and D. Habs, Generating positrons with femtosecond-laser pulses, Appl. Phys. Lett. 77, 2662 (2000).

[38] H. Chen, S. C. Wilks, J. D. Bonlie, E. P. Liang, J. Myatt, D. F. Price, D. D. Meyerhofer, and P. Beiersdorfer, Relativistic Positron Creation Using Ultraintense Short Pulse Lasers, Phys. Rev. Lett. 102, 105001 (2009).

[39] G. Sarri, K. Poder, J. Cole, W. Schumaker, A. Di Piazza, B. Reville, D. Doria, B. Dromey, L. Gizzi, A. Green, G. Grittani, S. Kar, C. H. Keitel, K. Krushelnick, S. Kushel, S. Mangles, Z. Najmudin, A. G. R. Thomas, M. Vargas, and M. Zepf, Generation of a neutral, high-density electron-positron plasma in the laboratory, Nat. Commun. 6, 6747 (2015).

[40] G. J. Williams, D. Barnak, G. Fiksel, A. Hazi, S. Kerr, C. Krauland, A. Link, M. J.-E. Manuel, S. R. Nagel, J. Park, J. Peebles, B. B. Pollock, F. N. Beg, R. Betti, and H. Chen, Target material dependence of positron generation from high intensity laser-matter interactions, Phys. Plasmas 23, 123109 (2016).

[41] J. H. Sung, H. W. Lee, J. Y. Yoo, J. W. Yoon, C. W. Lee, J. M. Yang, Y. J. Son, Y. H. Jang, S. K. Lee, and C. H. Nam, 4.2 PW, 20 fs Ti:sapphire laser at 0.1 Hz, Opt. Lett. 42, 2058 (2017).

[42] X. Zeng, K. Zhou, Y. Zuo, Q. Zhu, J. Su, X. Wang, X. Wang, X. Huang, X. Jiang, D. Jiang, Y. Guo, N. Xie, S. Zhou, Z. $\mathrm{Wu}, \mathrm{J} . \mathrm{Mu}, \mathrm{H}$. Peng, and F. Jing, Multi-petawatt laser facility fully based on optical parametric chirped-pulse amplification, Opt. Lett. 42, 2014 (2017).

[43] S. Weber, S. Bechet, S. Borneis, L. Brabec, M. Bučka, E. Chacon-Golcher, M. Ciappina, M. DeMarco, A. Fajstavr, K. Falk, E.-R. Garcia, J. Grosz, Y.-J. Gu, J.-C. Hernandez, M. Holec, P. Janečka, M. Jantač, M. Jirka, H. Kadlecova, D. Khikhlukha, O. Klimo, G. Korn, D. Kramer, D. Kumar, T. Lastovička, P. Lutoslawski, L. Morejon, V. Olšovcová, M. Rajdl, O. Renner, B. Rus, S. Singh, M. Šmid, M. Sokol, R. Versaci, R. Vrána, M. Vranic, J. Vyskočil, A. Wolf, and Q. Yu, P3: An installation for high-energy density plasma physics and ultra-high intensity laser-matter interaction at ELI-Beamlines, Matter Radiat. Extremes 2, 149 (2017).

[44] D. Papadopoulos, J. Zou, C. Le Blanc, G. Chériaux, P. Georges, F. Druon, G. Mennerat, P. Ramirez, L. Martin, A. Fréneaux, A. Beluze, N. Lebas, P. Monot, F. Mathieu, and P. Audebert, The APOLLON 10 pw laser: Experimental and theoretical investigation of the temporal characteristics, High Power Laser Sci. Eng. 4, e34 (2016).

[45] D. N. Papadopoulos, J. P. Zou, C. L. Blanc, L. Ranc, F. Druon, L. Martin, A. Fréneaux, A. Beluze, N. Lebas, M. Chabanis, C. Bonnin, J. B. Accary, B. L. Garrec, F. Mathieu, and P. Audebert, First commissioning results of the APOLLON laser on the $1 \mathrm{pw}$ beam line, in Conference on Lasers and ElectroOptics (Optical Society of America, Washington, DC, 2019), p. STu3E.4.

[46] A. Zhidkov, J. Koga, A. Sasaki, and M. Uesaka, Radiation Damping Effects on the Interaction of Ultraintense Laser Pulses with an Overdense Plasma, Phys. Rev. Lett. 88, 185002 (2002).

[47] J. Koga, Integration of the Lorentz-Dirac equation: Interaction of an intense laser pulse with high-energy electrons, Phys. Rev. E 70, 046502 (2004).

[48] T. Erber, High-energy electromagnetic conversion processes in intense magnetic fields, Rev. Mod. Phys. 38, 626 (1966).
[49] J. G. Kirk, A. R. Bell, and I. Arka, Pair production in counterpropagating laser beams, Plasma Phys. Control. Fusion 51, 085008 (2009).

[50] A. Di Piazza, C. Müller, K. Z. Hatsagortsyan, and C. H. Keitel, Extremely high-intensity laser interactions with fundamental quantum systems, Rev. Mod. Phys. 84, 1177 (2012).

[51] S. V. Popruzhenko, T. V. Liseykina, and A. Macchi, Efficiency of radiation friction losses in laser-driven 'hole boring' of dense targets, New J. Phys. 21, 033009 (2019).

[52] D. J. Stark, T. Toncian, and A. V. Arefiev, Enhanced Multi$\mathrm{MeV}$ Photon Emission by a Laser-Driven Electron Beam in a Self-Generated Magnetic Field, Phys. Rev. Lett. 116, 185003 (2016).

[53] F. Del Gaudio, T. Grismayer, R. A. Fonseca, W. B. Mori, and L. O. Silva, Bright $\gamma$ rays source and nonlinear breit-wheeler pairs in the collision of high density particle beams, Phys. Rev. Accel. Beams 22, 023402 (2019).

[54] K. Ta Phuoc, S. Corde, C. Thaury, V. Malka, A. Tafzi, J. P. Goddet, R. C. Shah, S. Sebban, and A. Rousse, All-optical Compton gamma-ray source, Nat. Photon. 6, 308 (2012).

[55] G. Sarri, D. J. Corvan, W. Schumaker, J. M. Cole, A. Di Piazza, H. Ahmed, C. Harvey, C. H. Keitel, K. Krushelnick, S. P. D. Mangles, Z. Najmudin, D. Symes, A. G. R. Thomas, M. Yeung, Z. Zhao, and M. Zepf, Ultrahigh Brilliance Multi$\mathrm{MeV} \gamma$-Ray Beams from Nonlinear Relativistic Thomson Scattering, Phys. Rev. Lett. 113, 224801 (2014).

[56] J. M. Cole, K. T. Behm, E. Gerstmayr, T. G. Blackburn, J. C. Wood, C. D. Baird, M. J. Duff, C. Harvey, A. Ilderton, A. S. Joglekar, K. Krushelnick, S. Kuschel, M. Marklund, P. McKenna, C. D. Murphy, K. Poder, C. P. Ridgers, G. M. Samarin, G. Sarri, D. R. Symes, A. G. R. Thomas, J. Warwick, M. Zepf, Z. Najmudin, and S. P. D. Mangles, Experimental Evidence of Radiation Reaction in the Collision of a HighIntensity Laser Pulse with a Laser-Wakefield Accelerated Electron Beam, Phys. Rev. X 8, 011020 (2018).

[57] K. Poder, M. Tamburini, G. Sarri, A. Di Piazza, S. Kuschel, C. D. Baird, K. Behm, S. Bohlen, J. M. Cole, D. J. Corvan, M. Duff, E. Gerstmayr, C. H. Keitel, K. Krushelnick, S. P. D. Mangles, P. McKenna, C. D. Murphy, Z. Najmudin, C. P. Ridgers, G. M. Samarin, D. R. Symes, A. G. R. Thomas, J. Warwick, and M. Zepf, Experimental Signatures of the Quantum Nature of Radiation Reaction in the Field of an Ultraintense Laser, Phys. Rev. X 8, 031004 (2018).

[58] T. G. Blackburn, C. P. Ridgers, J. G. Kirk, and A. R. Bell, Quantum Radiation Reaction in Laser-Electron-Beam Collisions, Phys. Rev. Lett. 112, 015001 (2014).

[59] M. Vranic, J. L. Martins, J. Vieira, R. A. Fonseca, and L. O. Silva, All-Optical Radiation Reaction at $10^{21} \mathrm{~W} / \mathrm{cm}^{2}$, Phys. Rev. Lett. 113, 134801 (2014).

[60] C. P. Ridgers, T. G. Blackburn, D. Del Sorbo, L. E. Bradley, C. Slade-Lowther, C. D. Baird, S. P. D. Mangles, P. McKenna, M. Marklund, C. D. Murphy, and A. G. R. Thomas, Signatures of quantum effects on radiation reaction in laser-electron-beam collisions, J. Plasma Phys. 83, 715830502 (2017).

[61] I. V. Sokolov, N. M. Naumova, J. A. Nees, G. A. Mourou, and V. P. Yanovsky, Dynamics of emitting electrons in strong laser fields, Phys. Plasmas 16, 093115 (2009).

[62] S. V. Bulanov, T. Z. Esirkepov, M. Kando, J. K. Koga, and S. S. Bulanov, Lorentz-Abraham-Dirac versus Landau-Lifshitz radiation friction force in the ultrarelativistic electron 
interaction with electromagnetic wave (exact solutions), Phys. Rev. E 84, 056605 (2011).

[63] F. Mackenroth, N. Neitz, and A. Di Piazza, Novel aspects of radiation reaction in the ultrarelativistic quantum regime, Plasma Phys. Control. Fusion 55, 124018 (2013).

[64] A. Di Piazza, M. Tamburini, S. Meuren, and C. H. Keitel, Implementing nonlinear Compton scattering beyond the localconstant-field approximation, Phys. Rev. A 98, 012134 (2018).

[65] N. Neitz and A. Di Piazza, Stochasticity Effects in Quantum Radiation Reaction, Phys. Rev. Lett. 111, 054802 (2013).

[66] C. N. Harvey, A. Gonoskov, A. Ilderton, and M. Marklund, Quantum Quenching of Radiation Losses in Short Laser Pulses, Phys. Rev. Lett. 118, 105004 (2017).

[67] A. Gonoskov, A. Bashinov, I. Gonoskov, C. Harvey, A. Ilderton, A. Kim, M. Marklund, G. Mourou, and A. Sergeev, Anomalous Radiative Trapping in Laser Fields of Extreme Intensity, Phys. Rev. Lett. 113, 014801 (2014).

[68] T. Grismayer, M. Vranic, J. L. Martins, R. A. Fonseca, and L. O. Silva, Seeded QED cascades in counterpropagating laser pulses, Phys. Rev. E 95, 023210 (2017).

[69] C. S. Brady, C. P. Ridgers, T. D. Arber, and A. R. Bell, Synchrotron radiation, pair production, and longitudinal electron motion during 10-100 PW laser solid interactions, Phys. Plasmas 21, 033108 (2014).

[70] E. N. Nerush, I. Y. Kostyukov, L. Ji, and A. Pukhov, Gammaray generation in ultrahigh-intensity laser-foil interactions, Phys. Plasmas 21, 013109 (2014).

[71] H. Y. Wang, B. Liu, X. Q. Yan, and M. Zepf, Gamma-ray emission in near critical density plasmas at laser intensities of $10^{21} \mathrm{~W} / \mathrm{cm}^{2}$, Phys. Plasmas 22, 033102 (2015).

[72] H. X. Chang, B. Qiao, Y. X. Zhang, Z. Xu, W. P. Yao, C. T. Zhou, and X. T. He, Ultraintense laser absorption and $\gamma$-ray synchrotron radiation in near critical density plasmas, Phys. Plasmas 24, 043111 (2017).

[73] R. R. Pandit and Y. Sentoku, Higher order terms of radiative damping in extreme intense laser-matter interaction, Phys. Plasmas 19, 073304 (2012).

[74] R. Ward and N. J. Sircombe, Fast particle Bremsstrahlung effects in the PIC code EPOCH: Enhanced diagnostics for laser-solid interaction modelling, Central Laser Facility Annual Report 2013-2014, 2014.

[75] F. Wan, C. Lv, M. Jia, H. Sang, and B. Xie, Photon emission by bremsstrahlung and nonlinear compton scattering in the interaction of ultraintense laser with plasmas, Eur. Phys. J. D 71, 236 (2017).

[76] J. Vyskočil, O. Klimo, and S. Weber, Simulations of bremsstrahlung emission in ultra-intense laser interactions with foil targets, Plasma Phys. Control. Fusion 60, 054013 (2018).

[77] D. Wu, X. T. He, W. Yu, and S. Fritzsche, Particle-in-cell simulations of laser-plasma interactions at solid densities and relativistic intensities: The role of atomic processes, High Power Laser Sci. Eng. 6, e50 (2018).

[78] B. Martinez, M. Lobet, R. Duclous, E. d'Humières, and L. Gremillet, High-energy radiation and pair production by Coulomb processes in particle-in-cell simulations, Phys. Plasmas 26, 103109 (2019).

[79] J. Vyskočil, E. Gelfer, and O. Klimo, Inverse Compton scattering from solid targets irradiated by ultra-short laser pulses in the $10^{22}-10^{23} \mathrm{~W} / \mathrm{cm}^{2}$ regime, Plasma Phys. Control. Fusion 62, 064002 (2020).

[80] E. Lefebvre, N. Cochet, S. Fritzler, V. Malka, M.-M. Aléonard, J.-F. Chemin, S. Darbon, L. Disdier, J. Faure, A. Fedotoff, O. Landoas, G. Malka, V. Méot, P. Morel, M. Rabec LeGloahec, A. Rouyer, C. Rubbelynck, V. Tikhonchuk, R. Wrobel, P. Audebert, and C. Rousseaux, Electron and photon production from relativistic laser plasma interactions, Nucl. Fus. 43, 629 (2003).

[81] M. Lobet, E. d'Humières, M. Grech, C. Ruyer, X. Davoine, and L. Gremillet, Modeling of radiative and quantum electrodynamics effects in PIC simulations of ultra-relativistic laser-plasma interaction, J. Phys.: Conf. Ser. 688, 012058 (2016).

[82] B. Martinez, E. d'Humières, and L. Gremillet, Synchrotron emission from nanowire array targets irradiated by ultraintense laser pulses, Plasma Phys. Controlled Fusion 60, 074009 (2018).

[83] F. Pérez, L. Gremillet, A. Decoster, M. Drouin, and E. Lefebvre, Improved modeling of relativistic collisions and collisional ionization in particle-in-cell codes, Phys. Plasmas 19, 083104 (2012).

[84] R. Duclous, J. G. Kirk, and A. R. Bell, Monte Carlo calculations of pair production in high-intensity laser-plasma interactions, Plasma Phys. Controlled Fusion 53, 015009 (2011).

[85] S. M. Weng, P. Mulser, and Z. M. Sheng, Relativistic critical density increase and relaxation and high-power pulse propagation, Phys. Plasmas 19, 022705 (2012).

[86] A. Debayle, F. Mollica, B. Vauzour, Y. Wan, A. Flacco, V. Malka, X. Davoine, and L. Gremillet, Electron heating by intense short-pulse lasers propagating through near-critical plasmas, New J. Phys. 19, 123013 (2017).

[87] C. S. Brady, C. P. Ridgers, T. D. Arber, A. R. Bell, and J. G. Kirk, Laser Absorption in Relativistically Underdense Plasmas by Synchrotron Radiation, Phys. Rev. Lett. 109, 245006 (2012).

[88] D. Bauer and P. Mulser, Vacuum heating versus skin layer absorption of intense femtosecond laser pulses, Phys. Plasmas 14, 023301 (2007).

[89] J. May, J. Tonge, F. Fiuza, R. A. Fonseca, L. O. Silva, C. Ren, and W. B. Mori, Mechanism of generating fast electrons by an intense laser at a steep overdense interface, Phys. Rev. E 84, 025401(R) (2011).

[90] A. Debayle, J. Sanz, L. Gremillet, and K. Mima, Toward a selfconsistent model of the interaction between an ultra-intense, normally incident laser pulse with an overdense plasma, Phys. Plasmas 20, 053107 (2013).

[91] A. A. Gonoskov, A. V. Korzhimanov, A. V. Kim, M. Marklund, and A. M. Sergeev, Ultrarelativistic nanoplasmonics as a route towards extreme-intensity attosecond pulses, Phys. Rev. E 84, 046403 (2011).

[92] L. O. Silva, M. Marti, J. R. Davies, R. A. Fonseca, C. Ren, F. S. Tsung, and W. B. Mori, Proton Shock Acceleration in Laser-Plasma Interactions, Phys. Rev. Lett. 92, 015002 (2004).

[93] A. Macchi, M. Borghesi, and M. Passoni, Ion acceleration by superintense laser-plasma interaction, Rev. Mod. Phys. 85, 751 (2013). 
[94] R. Nuter, L. Gremillet, E. Lefebvre, A. Lévy, T. Ceccotti, and P. Martin, Field ionization model implemented in Particle In Cell code and applied to laser-accelerated carbon ions, Phys. Plasmas 18, 033107 (2011).

[95] E. d'Humières, E. Lefebvre, L. Gremillet, and V. Malka, Proton acceleration mechanisms in high-intensity laser interaction with thin foils, Phys. Plasmas 12, 062704 (2005).

[96] T. Esirkepov, M. Yamagiwa, and T. Tajima, Laser IonAcceleration Scaling Laws Seen in Multiparametric ParticleIn-Cell Simulations, Phys. Rev. Lett. 96, 105001 (2006).

[97] A. V. Brantov, E. A. Govras, V. Y. Bychenkov, and W. Rozmus, Ion energy scaling under optimum conditions of laser plasma acceleration from solid density targets, Phys. Rev. ST Accel. Beams 18, 021301 (2015).

[98] J. Ferri, E. Siminos, L. Gremillet, and T. Fülöp, Effects of oblique incidence and colliding pulses on laser-driven proton acceleration from relativistically transparent ultrathin targets, J. Plasma Phys. 86, 905860505 (2020).

[99] V. A. Vshivkov, N. M. Naumova, F. Pegoraro, and S. V. Bulanov, Nonlinear electrodynamics of the interaction of ultra-intense laser pulses with a thin foil, Phys. Plasmas 5, 2727 (1998).

[100] A. Macchi, Theory of light sail acceleration by intense lasers: an overview, High Power Laser Sci. Eng. 2, e10 (2014).

[101] X. Q. Yan, C. Lin, Z. M. Sheng, Z. Y. Guo, B. C. Liu, Y. R. Lu, J. X. Fang, and J. E. Chen, Generating High-Current Monoenergetic Proton Beams by a Circularly Polarized Laser Pulse in the Phase-Stable Acceleration Regime, Phys. Rev. Lett. 100, 135003 (2008).

[102] C. Quigg, Electron-ion bremsstrahlung from an extreme relativistic plasma, Astrophys. J. 151, 1187 (1968).

[103] C. D. Dermer and G. Menon, High Energy Radiation from Black Holes: Gamma Rays, Cosmic Rays, and Neutrinos. Princeton Series in Astrophysics (Princeton University Press, Princeton, NJ, 2009).

[104] J. Polz, A. P. L. Robinson, A. Kalinin, G. A. Becker, R. A. C. Fraga, M. Hellwing, M. Hornung, S. Keppler, A. Kessler, D. Klöpfel, H. Liebetrau, F. Schorcht, J. Hein, M. Zepf, R. E. Grisenti, and M. C. Kaluza, Efficient laser-driven proton acceleration from a cryogenic solid hydrogen target, Sci. Rep. 9, 16534 (2019). 